\title{
IL-1R8 is a checkpoint in NK cells regulating anti-tumour and anti-viral activity
}

Martina Molgora ${ }^{1 *}$, Eduardo Bonavita ${ }^{1 * \dagger}+$, Andrea Ponzetta ${ }^{1}$, Federica Riva ${ }^{2}$, Marialuisa Barbagallo $^{1}$, Sébastien Jaillon ${ }^{1,3}$, Branka Popović $^{4}$, Giovanni Bernardini ${ }^{5,6}$, Elena Magrini ${ }^{1}$, Francesca Gianni ${ }^{1}$, Santiago Zelenay ${ }^{7}$, Stipan Jonjić ${ }^{4}$, Angela Santoni ${ }^{5,6}$, Cecilia Garlanda ${ }^{1,3}$ \& Alberto Mantovani ${ }^{1,3,8}$

Interleukin-1 receptor 8 (IL-1R8, also known as single immunoglobulin IL-1R-related receptor, SIGIRR, or TIR8) is a member of the IL-1 receptor (ILR) family with distinct structural and functional characteristics, acting as a negative regulator of ILR and Toll-like receptor (TLR) downstream signalling pathways and inflammation ${ }^{1}$. Natural killer (NK) cells are innate lymphoid cells which mediate resistance against pathogens and contribute to the activation and orientation of adaptive immune responses ${ }^{2-4}$. NK cells mediate resistance against haematopoietic neoplasms but are generally considered to play a minor role in solid tumour carcinogenesis ${ }^{5-7}$. Here we report that IL-1R8 serves as a checkpoint for NK cell maturation and effector function. Its genetic blockade unleashes NK-cell-mediated resistance to hepatic carcinogenesis, haematogenous liver and lung metastasis, and cytomegalovirus infection.

Several lines of evidence suggest that IL-1R8 interferes with the association of TIR module-containing adaptor molecules with signalling receptor complexes of the ILR or TLR family, tuning downstream signalling, thus negatively controlling inflammatory and immune responses and $\mathrm{T}$ helper cell polarization and functions ${ }^{1,8}$. Moreover, IL-1R8 is the co-receptor of IL-1R5/IL-18R $\alpha$ for IL-37, and is required for the anti-inflammatory activity of this human cytokine ${ }^{9}$. Deregulated activation by ILR or TLR ligands in IL-1R8-deficient mice has been associated with exacerbated inflammation and immunopathology, including selected cancers, or autoimmune diseases ${ }^{10}$.

IL-1R8 is widely expressed ${ }^{10}$. However, we found strikingly high levels of IL-1R8 mRNA and protein in human NK cells, compared with other circulating leukocytes and monocyte-derived macrophages (Fig. 1a and Extended Data Fig. 1a). IL1R8 mRNA levels increased during NK cell maturation ${ }^{11}$ (Extended Data Fig. 1b) and surface protein expression mirrored transcript levels (Fig. $1 \mathrm{~b}$ and Extended Data Fig. 1c). IL-1R8 expression was detected at a low level in bone marrow pluripotent haematopoietic stem cells and NK cell precursors, and was selectively upregulated in mature NK cells but not in $\mathrm{CD}^{+}$ lymphocytes (Extended Data Fig. 1d).

Mouse NK cells expressed significantly higher levels of Il1r $8 \mathrm{mRNA}$ compared with other leukocytes (Fig. 1c) and other ILRs (Extended Data Fig. 1e, f). In line with the results obtained in human NK cells, the Il1r 8 mRNA level increased during the four-stage developmental transition from $\mathrm{CD} 11 \mathrm{~b}^{\text {low }} \mathrm{CD} 27^{\text {low }}$ to $\mathrm{CD} 11 \mathrm{~b}^{\text {high }} \mathrm{CD} 27^{\text {low }}$ (ref. 12) (Fig. 1d and Extended Data Fig. 1g).

To assess the role of IL-1R8 in NK cells, we took advantage of IL-1R8deficient mice. Among $\mathrm{CD} 45^{+}$cells, the NK cell frequency and absolute numbers were significantly higher in peripheral blood of $111 r 8^{-/-}$ compared with Ill $r 8^{+/+}$mice, and slightly increased in liver and spleen. (Fig. 2a, b). In addition, the frequency of the CD11b ${ }^{\text {high }} \mathrm{CD} 27^{\text {low }}$ and
$\mathrm{KLRG}^{+}$mature subsets were significantly higher in $I l 1 r 8^{-1-}$ mice than $I l 1 r 8^{+/+}$mice in bone marrow, spleen and blood, indicating a more mature phenotype of NK cells ${ }^{13}$ (Fig. 2c, d and Extended Data Fig. 2a, b).

The enhanced NK cell maturation in $I l 1 r 8^{-1-}$ mice occurred already at 2 and 3 weeks of age, whereas the frequency of NK precursors was similar in $1 l 1 r 8^{-1-}$ and $I l 1 r 8^{+/+}$bone marrow, indicating that IL-1R8 regulated early events in NK cell differentiation, but did not affect the development of NK cell precursors ${ }^{12}$ (Extended Data Fig. 2c-e).

We next investigated whether IL-1R8 affected NK cell function. The expression of the activating receptors NKG2D, DNAM-1 and $\mathrm{Ly} 49 \mathrm{H}$ was significantly upregulated in peripheral blood $I l 1 r 8^{-1-} \mathrm{NK}$ cells (Extended Data Fig. 2f). Interferon- $\gamma($ IFN $\gamma$ ) and granzyme B production and FasL expression were more sustained in IL-1R8deficient NK cells upon ex vivo stimulation in the presence of IL-18 (Fig. 2e-g and Extended Data Fig. 2g). The frequency of IFN $\gamma^{+} \mathrm{NK}$ cells was higher in $I l 1 r 8^{-1-}$ total NK cells and in all NK cell subsets. Thus, IFN $\gamma$ production was enhanced independently of the NK cell maturation state. Analysis of competitive bone marrow chimaeras revealed that IL-1R8 regulates NK cell differentiation in a cellautonomous way (Extended Data Fig. 2h-k). Along the same line, co-culture experiments of NK cells with lipopolysaccharide (LPS)- or CpG-primed dendritic cells showed that $I l 118^{-I-} \mathrm{NK}$ cells produced higher IFN $\gamma$ levels irrespective of the dendritic cell genotype (Extended Data Fig. 2l).

IL-18 is a member of the IL- 1 family, which plays an important role in NK cell differentiation and function ${ }^{1,14}$. Enhanced NK cell maturation and effector function in $I l 1 r 8^{-1-}$ mice was abolished by IL-18 blockade or genetic deficiency but unaffected by IL-1R1-deficiency (Fig. 2h, i and Extended Data Fig. 3a, b). Co-housing and antibiotic treatment had no impact, thus excluding a role of microbiota ${ }^{15}$ in the phenotype of Il1r8 $8^{-l-}$ mice (Extended Data Fig. 3c, d).

The results reported above suggested that IL-1R8 regulated the IL-18 signalling pathway in NK cells and, indeed, an increased phospho-IRAK4/IRAK4 ratio was induced by IL-18 in $I l 1 r 8^{-/-} \mathrm{NK}$ cells compared with wild-type NK cells, indicating unleashed early signalling downstream of MyD88 and myddosome formation (Fig. 2j), consistent with the proposed molecular mode of action of IL-1R8 (refs $1,9,16$ ). Indeed, by stimulated emission depletion (STED) microscopy, we observed clustering of IL-1R8 and IL-18R $\alpha$ (Extended Data Fig. 3e), in line with previous studies ${ }^{9}$. IL-1R8-deficiency also led to enhanced IL-18-dependent phosphorylation of S6 and JNK in NK cells, suggesting that IL-1R8 inhibited IL-18-dependent activation of the mTOR and JNK pathways (Fig. 2j), which control NK cell metabolism, differentiation and activation ${ }^{17,18}$. 

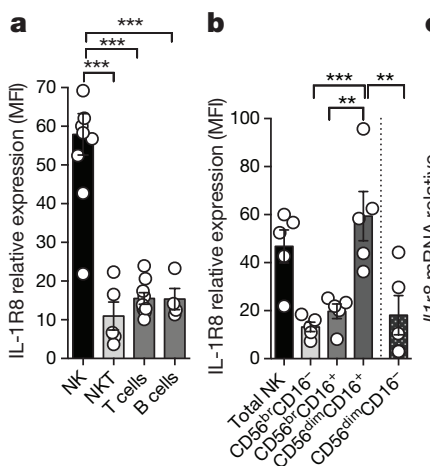

c

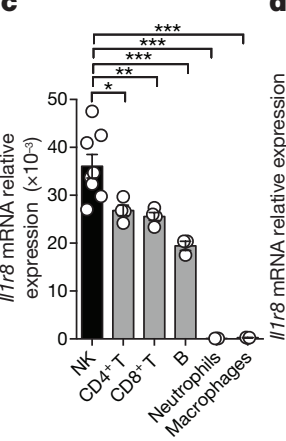

\section{d}

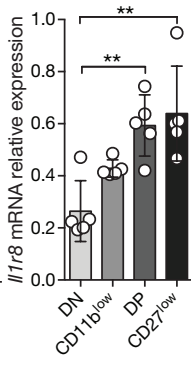

Figure 1 | Expression of IL-1R8 in human and mouse NK cells. a, b, IL-1R8 protein expression in human primary NK cells and other leukocytes (a) and NK cell maturation stages (b). MFI, mean fluorescence intensity. c, d, Il1r 8 mRNA expression in mouse primary NK cells and other leukocytes (c) and in sorted splenic NK cell subsets $(\mathbf{d}) . * P<0.05$, $* * P<0.01, * * * P<0.001$, one-way analysis of variance (ANOVA). Mean \pm s.e.m.

To obtain a deeper insight into the impact of IL-1R8 deficiency on NK cell function and on the response to IL-18, RNA sequencing (RNAseq) analysis was conducted. IL-1R8 deficiency had a profound impact on the resting transcriptional profile of NK cells and on top on responsiveness to IL-18 (Fig. 2k, Extended Data Fig. 4a and Supplementary Table 1). The profile of IL-1R8-deficient cells includes activation pathways (for example, MAPK), adhesion molecules involved in cell-tocell interactions and cytotoxicity (ICAM-1), and increased production of selected chemokines (CCL4). The last of these may represent an NK-cell-based amplification loop of leukocyte recruitment, including NK cells themselves.

To investigate the role of IL-1R8 in human NK cells (Fig. 1a, b), we first retrospectively analysed its expression in relation to responsiveness to a combination of IL-18 and IL-12 in normal donors. We observed an inverse correlation between IL-1R8 levels and IFN $\gamma$ production by peripheral blood NK cells $\left(r^{2}=0.7969, P=0.0012\right)$ (Fig. 21). In addition, IL-1R8 partial silencing in peripheral blood NK cells with small interfering RNA (siRNA) was associated with a significant increase in IFN $\gamma$ production (Fig. $2 \mathrm{~m}$ ) and upregulation of CD69 expression (not shown). These results suggest that in human NK cells, as in mouse counterparts, IL-1R8 serves as a negative regulator of activation and that its inactivation unleashes human NK-cell effector function.

To assess the actual relevance of IL-1R8-mediated regulation of NK cells, anticancer and anti-viral resistance were examined. The liver is characterized by a high frequency of NK cells ${ }^{19}$. Therefore we focused on liver carcinogenesis. In a model of diethylnitrosamine-induced

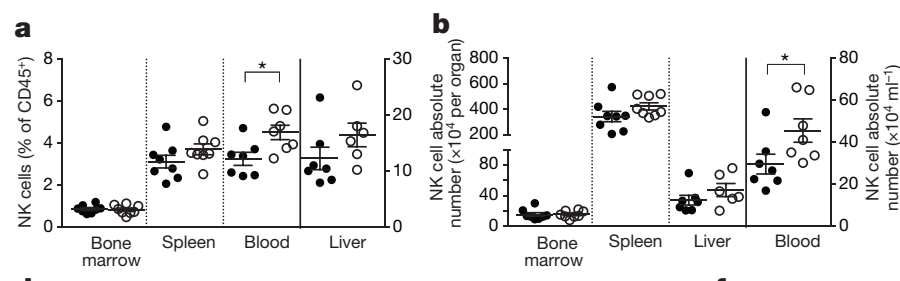

c
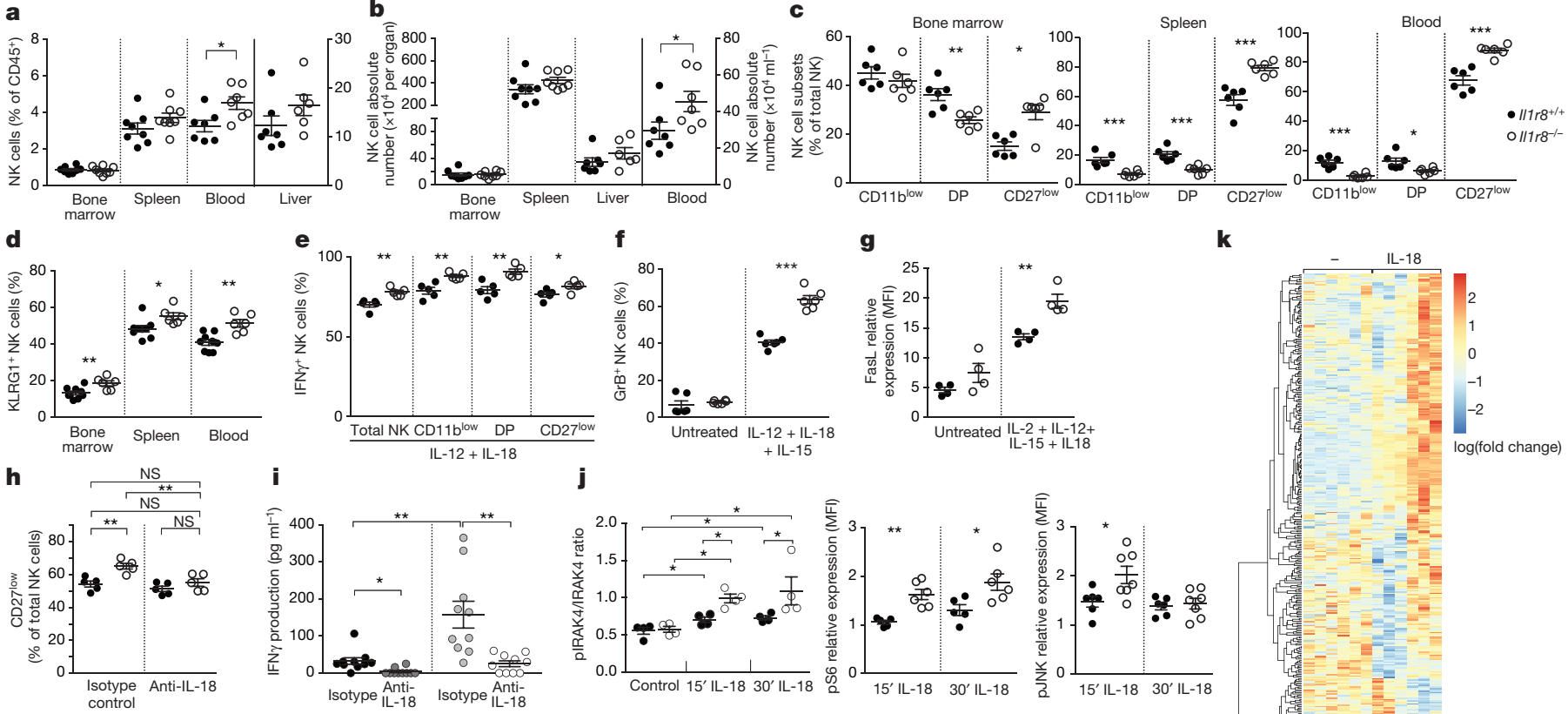

$\mathbf{k}$ $\begin{array}{llllll}\text { NK } & 111 r 8 & +/+ & +/+ & -/- & -/- \\ \text { BMDCs } & 111 r 8 & +/+ & +/+ & +/+ & +/+ \\ \text { (CpG-primed) } & & & & \end{array}$

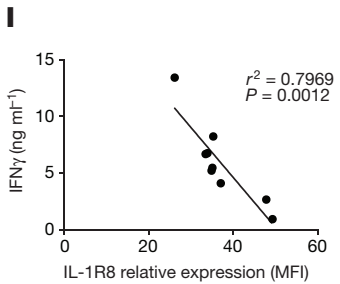

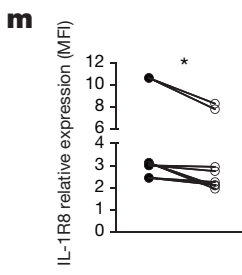

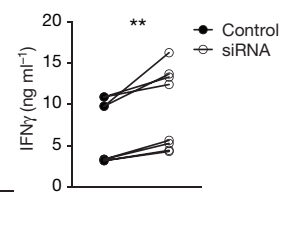

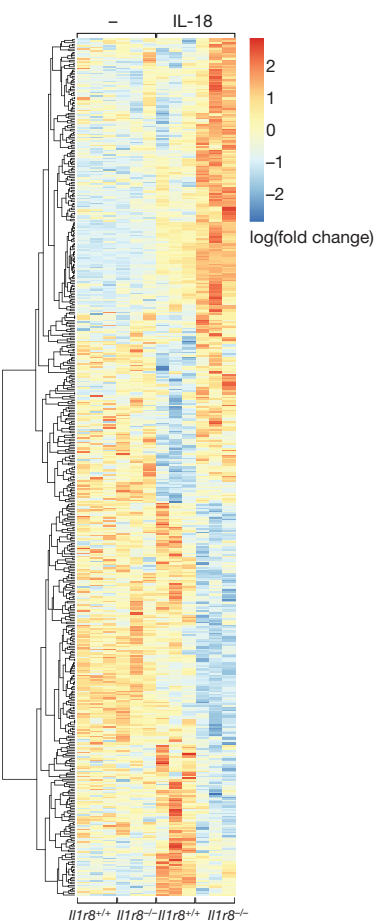

Figure $2 \mid$ NK cell differentiation and function in IL-1R8-deficient mice. $\mathbf{a}, \mathbf{b}, \mathrm{NK}$ cell frequency and absolute number among leukocytes in $111 \mathrm{r} 8^{+/+}$ and $I l 1 r 8^{-1-}$ mice. $\mathbf{c}$, d, NK cell subsets (c) and KLRG1 $1^{+}$NK cells (d). $\mathbf{e}-\mathbf{g}$, IFN $\gamma(\mathbf{e})$, granzyme B (f) and FasL (g) expression in stimulated NK cells. h, Splenic CD27 $7^{\text {low }}$ NK cell frequency upon IL-18 in vivo depletion. i, IFN $\gamma$ production by $I l 1 r 8^{+/+}$and $I l 1 r 8^{-/-}$NK cells upon co-culture with CpG-primed Ill $r 8^{+/+}$dendritic cells and IL-18 blockade. j, IRAK4, S6 and JNK phosphorylation in NK cells upon stimulation with IL-18 for 15 or $30 \mathrm{~min}$. k, RNA-seq analysis of resting and IL-18-activated NK cells. Differentially expressed $(P<0.05)$ genes are shown. 1 , Correlation between IL-1R8 expression and IFN $\gamma$ production in human peripheral blood NK cells. $\mathbf{m}$, IL-1R8 expression and IFN $\gamma$ production in human NK cells 7 days after transfection with control siRNA or IL-1R8-specific siRNA in duplicate. $\mathbf{a}-\mathbf{l}$, $* P<0.05, * * P<0.01$, *** $P<0.001$ between selected relevant comparisons, two-tailed unpaired Student's $t$-test or MannWhitney $U$-test; $\mathbf{k}, r$ is Pearson's correlation coefficient. Mean \pm s.e.m. 


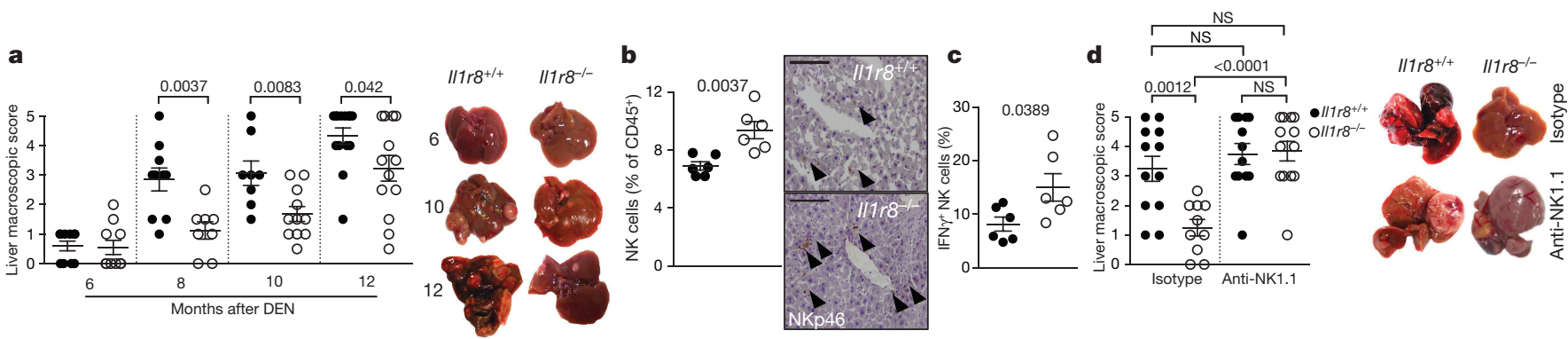

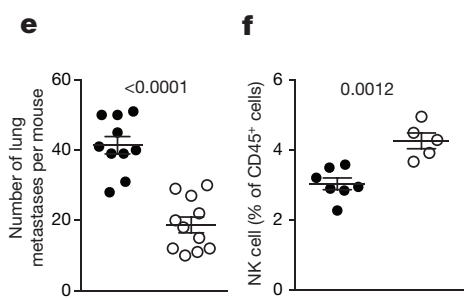

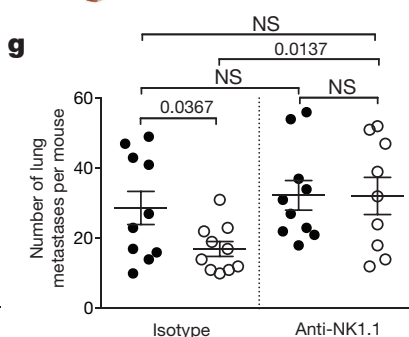

Figure 3 NK-cell-mediated protection against liver carcinogenesis and metastasis in IL-1R8-deficient mice. a, Macroscopic score of liver lesions in male Ill $1 \mathrm{r}^{+/+}$and $I l 1 r 8^{-l-}$ mice 6, 8, 10 and 12 months after diethylnitrosamine (DEN) injection. $P$ values are given at the tops of graphs. b. Frequency and representative histological quantification of NK cell infiltrate in liver of tumour-bearing mice (original magnification $20 \times$; scale bar, $100 \mu \mathrm{m}$ ). c, Frequency of IFN $\gamma^{+}$NK cells in liver of tumour-bearing mice. d, Macroscopic score of liver lesions in male mice

hepatocellular carcinoma, IL-1R8-deficient male and female mice ${ }^{20}$ were protected against the development of lesions, in terms of macroscopic number, size (Fig. 3a and Extended Data Fig. 5a, b) and histology (data not shown). The percentage and absolute number of NK cells, and the percentage of IFN $\gamma^{+} \mathrm{NK}$ cells, were higher in $I l 1 r 8^{-1-}$ hepatocellular carcinoma-bearing mice (Fig. 3b, c and Extended Data Fig. 5c). Finally, increased levels of cytokines involved in anti-tumour immunity (for example, IFN $\gamma$ ) and a reduction of pro-inflammatory cytokines associated with tumour promotion (IL-6, TNF, IL-13, CCL2, CXCL1) were observed (Extended Data Table 1). Most importantly, the depletion of NK cells abolished the protection against liver carcinogenesis observed in Illr8 ${ }^{-l-}$ mice (Fig. 3d and Extended Data Fig. 5d).

Evidence suggests that NK cells can inhibit haematogenous cancer metastasis $^{5}$. In a model of sarcoma (MN/MCA1) spontaneous lung metastasis, $I l 1 r^{-1-}$ mice showed a reduced number of haematogenous metastases, whereas primary tumour growth was unaffected (Fig. 3e and Extended Data Fig. 5e, f). The frequency of total and mature CD27 $7^{\text {low }}$ NK cells was higher in $I l 1 r 8^{-l-}$ lungs (Fig. $3 \mathrm{f}$ and data not shown). Assessment of lung metastasis at the time of euthanasia and in vivo imaging analysis (Fig. $3 g$ and Extended Data Fig. 5e) showed that the protection was completely abolished in NK-cell-depleted Il $1 r^{-1-}$ mice. In addition, IL-18 or IFN $\gamma$ neutralization abolished or markedly reduced the protection against metastasis observed in $I l 1 r 8^{-1-}$ mice (Extended Data Fig. 5g). In contrast, depletion of $\mathrm{CD}^{+} / \mathrm{CD}^{+}$cells or IL-17A, or deficiency of IL-1R1 (involved in Thelper 17 cell development), did not affect the phenotype (Extended Data Fig. 5h, i).

Liver metastasis is a major problem in the progression of colorectal cancer. We therefore assessed the potential of $I l 1 \mathrm{r}^{-1-} \mathrm{NK}$ cells to protect against liver metastasis using the MC38 colon carcinoma line ${ }^{21}$. As shown in Fig. 3h, Ill $18^{-1-}$ mice were protected against MC38 colon carcinoma liver metastasis. In addition, IL-18 genetic deficiency abrogated the protection against liver metastasis observed in $I l 1 r 8^{--}$mice (Extended Data Fig. 5j), thus indicating that the IL-1R8-dependent control of MC38-derived liver metastasis occurs through the IL-18/ IL-18R axis. To assess the primary role of $I l 1 \mathrm{r}^{-1-} \mathrm{NK}$ cells in the cancer protection, adoptive transfer was used (Extended Data Fig. 5k-m). Adoptive transfer of $I l 118^{+/+} \mathrm{NK}$ cells had no effect on lung and liver

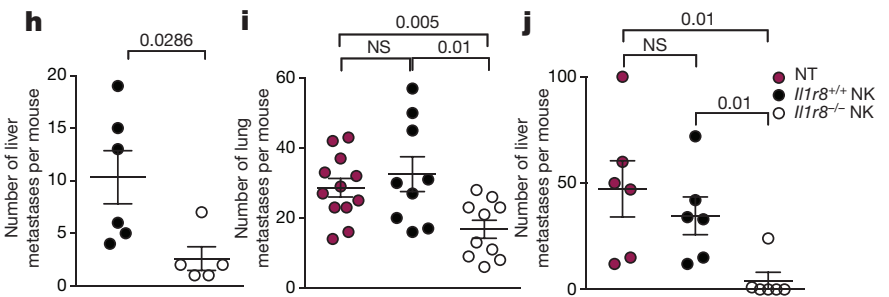

upon NK cell depletion. e, Number of spontaneous lung metastases. f, NK cell frequency in the lungs of MN/MCA1 tumour-bearing mice. g, Number of lung metastases in MN/MCA1 tumour-bearing mice upon NK cell depletion. h, Number of liver metastases in MC38 colon carcinoma-bearing mice. $\mathbf{i}, \mathbf{j}$, Number of lung (i) and liver (j) metastases of Ill $r 8^{+/+}$mice after adoptive transfer of $I l 1 r 8^{+/+}$and $I l 1 r 8^{-l-}$ NK cells. NT, not treated. a, d, Representative images of female livers are shown. $\mathbf{a}-\mathbf{j}$, Exact $P$ values are given.

metastasis. In contrast, adoptive transfer of $I l 1 r 8^{-1-} \mathrm{NK}$ cells significantly and markedly reduced the number and volume of lung and liver metastases (Fig. 3i, j and Extended Data Fig. 5n). Given the natural history and clinical challenges of colorectal cancer, this observation has potential translational implications. Thus, IL-1R8 genetic inactivation unleashes NK-cell-mediated resistance to carcinogenesis in the liver and amplifies the anti-metastatic potential of these cells in liver and lung in a NK-cell-autonomous manner.

Finally, we investigated whether IL-1R8 affects NK cell anti-viral activity, focusing on murine cytomegalovirus (MCMV) infection ${ }^{22}$. As shown in Fig. 4a, liver viral titres were lower in $I l 11 r^{-I-}$ than $I l 1 r 8^{+/+}$ mice, indicating that IL-1R8-deficiency was associated with a more efficient control of MCMV infection. The frequency of IFN $\gamma^{+} \mathrm{NK}$ cells and degranulation (that is, the frequency of $\mathrm{CD} 107 \mathrm{a}^{+} \mathrm{NK}$ cells) were significantly higher in the spleen and liver of $1 l 1 \mathrm{r}^{-1-}$ mice on day 1.5 after infection (Fig. 4b). On day 4.5 after infection, IFN $\gamma^{+}$and $\mathrm{CD} 107 \mathrm{a}^{+} \mathrm{NK}$ cells were strongly reduced, in both spleen and liver, as a consequence of better control of viral spread (Fig. 4b). Consistent with a more efficient control of the infection, reduced levels of proinflammatory cytokines were observed in $I l 1 \mathrm{r}^{-1-}$ mice (Extended Data Fig. 6a). NK-cell adoptive transfer experiments were performed in MCMV-infected newborn mice that still did not have mature NK cells ${ }^{12}$. As shown in Fig. 4c, the adoptive transfer of $I l 1 r 8^{-1-}$ NK cells conferred higher protection than $I l 1 r 8^{+/+}$NK cells, with, for instance, four out of nine mice having no detectable virus titre in the brain.

NK cells belong to the complex, diverse realm of innate lymphoid cells (ILCs) ${ }^{23}$. Human and mouse non-NK ILCs express IL-1R8 mRNA and protein (ref. 24, our unpublished data and C. Jandus, personal communication). Preliminary experiments were conducted to assess the role of IL-1R8 in ILC function. In the MCMV infection model, Ill $r 8^{-I-}$ ILC1 showed increased IFN $\gamma$ production, but represented a minor population compared with NK cells and one-thirtieth that of Ill $r 8^{-1-}$ IFN $\gamma$-producing cells (Fig. $4 \mathrm{~d}$ ); they are therefore unlikely to play a significant role in the phenotype. These results provide initial evidence that IL-1R8 has a regulatory function in ILCs. Further studies are required to assess its actual relevance in ILC diverse populations. Collectively, these results indicate that IL-1R8-deficient mice were 

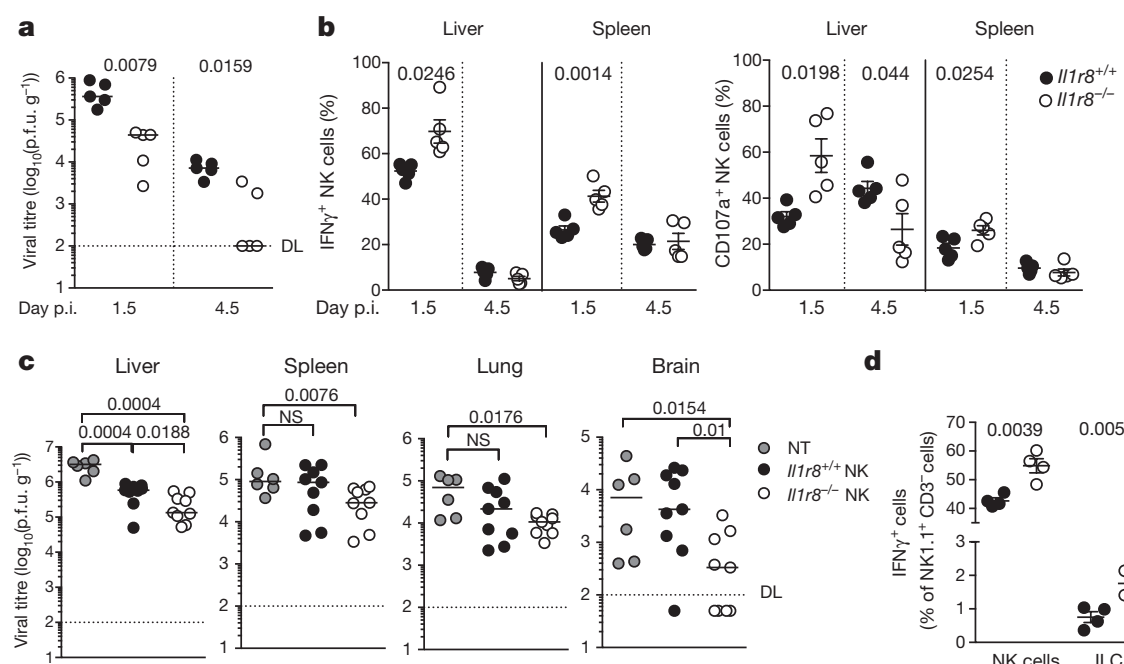

d

Figure $4 \mid$ NK-cell-mediated anti-viral resistance in IL-1R8-deficient mice. a, Viral titre in livers of $I l 1 r 8^{+/+}$and $I l 1 r 8^{-1-}$ infected mice. DL, detection limit. Day p.i., day post-infection; p.f.u., plaque-forming units. b, Frequency of IFN $\gamma^{+}$and CD107a ${ }^{+}$NK cells of infected mice. c, Viral titres in newborn wild-type mice upon adoptive transfer of

protected against MCMV infection and that protection was dependent on increased NK cell activation.

IL-1R8 deficiency was associated with exacerbated inflammatory and immune reactions under a variety of conditions ${ }^{1,10}$. NK cells engage in bidirectional interactions with macrophages, dendritic cells and other lymphocytes $3,4,25,26$. Therefore the role of NK cells in inflammatory and autoimmune conditions associated with IL-1R8 deficiency ${ }^{1,10}$ will need to be examined. IL-1R8-deficient mice show increased susceptibility to colitis and colitis-associated azoxymethane carcinogenesis ${ }^{27,28}$. The divergent impact on carcinogenesis of IL-1R8 deficiency in the intestine and liver is likely to reflect fundamental, tissue-dictated differences of immune mechanisms involved in carcinogenesis in these different anatomical sites. In particular, high numbers of NK cells are present in the liver ${ }^{19}$ and this physiological characteristic of this organ is likely to underlie this apparent divergence.

NK cells are generally not credited with playing a major role in the control of solid tumours ${ }^{6}$. Conversely there is evidence for a role of NK cells in the control of haematogenous lung metastasis ${ }^{5,29}$. The results presented here show that unleashing NK cells by genetic inactivation of IL-1R8 resulted in inhibition of liver carcinogenesis and protection against liver and lung metastasis. IL-1R8-deficient mice show exacerbated TLR and IL-1-driven inflammation ${ }^{10}$, and inflammation promotes liver carcinogenesis ${ }^{30}$. Therefore, our results are probably an underestimate of the potential of removal of the NK cell checkpoint IL-1R8 against liver primary and metastatic tumours. Thus, NK cells have the potential to restrain solid cancer and metastasis, provided critical, validated checkpoints such as IL-1R8 are removed and the tissue immunological landscape is taken into account.

Online Content Methods, along with any additional Extended Data display items and Source Data, are available in the online version of the paper; references unique to these sections appear only in the online paper.

\section{Received 22 September 2016; accepted 19 September 2017.} Published online 25 October 2017.

1. Garlanda, C., Dinarello, C. A. \& Mantovani, A. The interleukin-1 family: back to the future. Immunity 39, 1003-1018 (2013)

2. Di Santo, J. P. Natural killer cell developmental pathways: a question of balance. Annu. Rev. Immunol. 24, 257-286 (2006).

3. Vivier, E. et al. Innate or adaptive immunity? The example of natural killer cells. Science 331, 44-49 (2011)

4. Bellora, F. et al. Human NK cells and NK receptors. Immunol. Lett. 161, 168-173 (2014).

5. Guillerey, C., Huntington, N. D. \& Smyth, M. J. Targeting natural killer cells in cancer immunotherapy. Nat. Immunol. 17, 1025-1036 (2016).
Il $1 r 8^{+/+}$and Il1 $r 8^{-1-}$ NK cells (7 days after infection). d, Frequency of IFN $\gamma^{+}$cells in the liver of MCMV-infected mice. a-d, Exact $P$ values are given, determined by two-tailed Mann-Whitney $U$-test $(\mathbf{a}, \mathbf{c})$ or unpaired Student's $t$-test $(\mathbf{b}, \mathbf{d})$. Median $(\mathbf{a}, \mathbf{c})$; mean \pm s.e.m. $(\mathbf{b}, \mathbf{d})$. NT, not treated.

6. Stojanovic, A. \& Cerwenka, A. Natural killer cells and solid tumors. J. Innate Immun. 3, 355-364 (2011).

7. Gismondi, A., Stabile, H., Nisti, P. \& Santoni, A. Effector functions of natural killer cell subsets in the control of hematological malignancies. Front. Immunol. 6, 567 (2015).

8. Gulen, M. F. et al. The receptor SIGIRR suppresses Th17 cell proliferation via inhibition of the interleukin-1 receptor pathway and mTOR kinase activation. Immunity 32, 54-66 (2010).

9. Nold-Petry, C. A. et al. IL-37 requires the receptors IL-18R $\alpha$ and IL-1R8 (SIGIRR) to carry out its multifaceted anti-inflammatory program upon innate signal transduction. Nat. Immunol. 16, 354-365 (2015).

10. Garlanda, C., Riva, F., Bonavita, E. \& Mantovani, A. Negative regulatory receptors of the IL-1 family. Semin. Immunol. 25, 408-415 (2013).

11. Cooper, M. A. et al. Human natural killer cells: a unique innate immunoregulatory role for the CD56 ${ }^{\text {bright }}$ subset. Blood 97, 3146-3151 (2001).

12. Chiossone, L. et al. Maturation of mouse NK cells is a 4-stage developmental program. Blood 113, 5488-5496 (2009).

13. Kim, S. et al. In vivo developmental stages in murine natural killer cell maturation. Nat. Immunol. 3, 523-528 (2002).

14. Takeda, K. et al. Defective NK cell activity and Th1 response in IL-18-deficient mice. Immunity 8, 383-390 (1998).

15. Ganal, S. C. et al. Priming of natural killer cells by nonmucosal mononuclear phagocytes requires instructive signals from commensal microbiota. Immunity 37, 171-186 (2012).

16. Gong, J. et al. Inhibition of Toll-like receptors TLR4 and 7 signaling pathways by SIGIRR: a computational approach. J. Struct. Biol. 169, 323-330 (2010).

17. Marçais, A. et al. The metabolic checkpoint kinase mTOR is essential for IL-15 signaling during the development and activation of NK cells. Nat. Immunol. 15, 749-757 (2014).

18. Li, C. et al. JNK MAP kinase activation is required for MTOC and granule polarization in NKG2D-mediated NK cell cytotoxicity. Proc. Natl Acad. Sci. USA 105, 3017-3022 (2008).

19. Peng, H. \& Tian, Z. Re-examining the origin and function of liver-resident NK cells. Trends Immunol. 36, 293-299 (2015).

20. Naugler, W. E. et al. Gender disparity in liver cancer due to sex differences in MyD88-dependent IL-6 production. Science 317, 121-124 (2007).

21. Dupaul-Chicoine, J. et al. The NIrp3 inflammasome suppresses colorectal cancer metastatic growth in the liver by promoting natural killer cell tumoricidal activity. Immunity 43, 751-763 (2015).

22. Lisnić, B., Lisnić, V. J. \& Jonjić, S. NK cell interplay with cytomegaloviruses. Curr. Opin. Virol. 15, 9-18 (2015)

23. Eberl, G., Colonna, M., Di Santo, J. P. \& McKenzie, A. N. Innate lymphoid cells: a new paradigm in immunology. Science 348, aaa6566 (2015).

24. Shih, H. Y. et al. Developmental acquisition of regulomes underlies innate lymphoid cell functionality. Cell 165, 1120-1133 (2016).

25. Bellora, F. et al. M-CSF induces the expression of a membrane-bound form of $\mathrm{IL}-18$ in a subset of human monocytes differentiating in vitro toward macrophages. Eur. J. Immunol. 42, 1618-1626 (2012).

26. Martín-Fontecha, A. et al. Induced recruitment of NK cells to lymph nodes provides IFN-gamma for $\mathrm{T}(\mathrm{H}) 1$ priming. Nat. Immunol. 5, 1260-1265 (2004).

27. Garlanda, C. et al. Increased susceptibility to colitis-associated cancer of mice lacking TIR8, an inhibitory member of the interleukin-1 receptor family. Cancer Res. 67, 6017-6021 (2007) 
28. Xiao, H. et al. The Toll-interleukin-1 receptor member SIGIRR regulates colonic epithelial homeostasis, inflammation, and tumorigenesis. Immunity $\mathbf{2 6}$, 461-475 (2007).

29. Morvan, M. G. \& Lanier, L. L. NK cells and cancer: you can teach innate cells new tricks. Nat. Rev. Cancer 16, 7-19 (2016).

30. He, G. \& Karin, M. NF-kB and STAT3 - key players in liver inflammation and cancer. Cell Res. 21, 159-168 (2011).

Supplementary Information is available in the online version of the paper.

Acknowledgements We thank N. Polentarutti, G. Benigni, M. Erreni, F. Colombo, V. Juranić Lisnić and D. Kvestak and Computational and Molecular Biology CRUK MI core facilities for technical assistance, M. Nebuloni for hepatocellular carcinoma histology, A. Doni for STED images, and F. Ficara, R. Carriero and D. Mavilio for discussions. The contributions of the European Commission (ERC project PHII-669415; FP7 project 281608 TIMER; ESA/ITN, H2020-MSCAITN-2015-676129), Ministero dell'Istruzione, dell'Università e della Ricerca (MIUR) (project FIRB RBAP1 1H2R9), Associazione Italiana Ricerca sul Cancro (AIRC IG-19014 and AIRC 5x1000-9962), Fondazione CARIPLO (project 20150564), European Regional Development Fund (grant KK.01.1.1.01.0006, to S.J.) and the Italian Ministry of Health are gratefully acknowledged. M.M. received a
European Federation of Immunological Sciences short-term fellowship to perform viral infection experiments in the laboratory of S.Jo.

Author Contributions E.B. and M.M. played a key role in designing and conducting most experiments and drafted the manuscript. F.R., M.B., F.G. and E.M. provided technological support in in vivo experiments. A.P., S.Ja., B.P. and G.B. contributed to the experimental design and in vivo experiments. S.Z. contributed to RNA-seq analysis. S.Jo. and A.S. contributed to the experimental design and supervision of the study. C.G. and A.M. contributed to the experimental design and supervision of the study, and suggested the role of IL-1R8 as a novel checkpoint inhibitor of NK cells.

Author Information Reprints and permissions information is available at www.nature.com/reprints. The authors declare no competing financial interests. Readers are welcome to comment on the online version of the paper. Publisher's note: Springer Nature remains neutral with regard to jurisdictional claims in published maps and institutional affiliations. Correspondence and requests for materials should be addressed to C.G. (cecilia.garlanda@ humanitasresearch.it) or A.M. (alberto.mantovani@humanitasresearch.it).

Reviewer Information Nature thanks M. Karin, M. Smyth and the other anonymous reviewer(s) for their contribution to the peer review of this work. 


\section{METHODS}

Animals. All female and male mice used were on a C57BL/6J genetic background and were 8-12 weeks old, unless otherwise specified. Wild-type mice were obtained from Charles River Laboratories, Calco, Italy, or were littermates of $1 l 1 \mathrm{r}^{-I-}$ mice. IL-1R8-deficient mice were generated as described ${ }^{31}$. Ill $\mathrm{rl}^{-1-}$ mice were purchased from The Jackson Laboratory, Bar Harbour, Maine, USA. All colonies were housed and bred in the SPF animal facility of Humanitas Clinical and Research Center in individually ventilated cages. Ill $\mathrm{r}^{-1-} / \mathrm{Il} 1 \mathrm{r} \mathrm{B}^{-1-}$ mice were generated by crossing Ill $r 1^{-1-}$ and Ill $r 8^{-1-}$ mice. Ill $18^{-1-} / I l 11 \mathrm{r}^{-1-}$ were generated by crossing $I l 18^{-1-}$ and Ill $18^{-1-}$ mice. Mice were randomized on the basis of sex, age and weight.

Procedures involving animal handling and care conformed to protocols approved by the Humanitas Clinical and Research Center (Rozzano, Milan, Italy) in compliance with national (D.L. N.116, G.U., suppl. 40, 18-2-1992 and N. 26, G.U. March 4, 2014) and international law and policies (EEC Council Directive 2010/63/ EU, OJ L 276/33, 22-09-2010; National Institutes of Health Guide for the Care and Use of Laboratory Animals, US National Research Council, 2011). The study was approved by the Italian Ministry of Health (approval number 43/2012-B, issued on the 8 February 2012, and number 828/2015-PR, issued on the 7 August 2015). All efforts were made to minimize the number of animals used and their suffering. In most in vivo experiments, the investigators were unaware of the genotype of the experimental groups.

Human primary cells. Human peripheral mononuclear cells were isolated from peripheral blood of healthy donors, upon approval by the Humanitas Research Hospital Ethical Committee. Peripheral mononuclear cells were obtained through a Ficoll density gradient centrifugation (GE Healthcare Biosciences). NK cells were then purified by a negative selection, using a magnetic cell sorting technique according to the protocols given by the manufacturer (EasySep Human NK Cell Enrichment Kit, Stem Cell Technology). Human monocytes were obtained from peripheral blood of healthy donors by two-step gradient centrifugation, first by Ficoll and then by Percoll (65\% iso-osmotic; Pharmacia, Uppsala, Sweden). Residual $\mathrm{T}$ and $\mathrm{B}$ cells were removed from the monocyte fraction by plastic adherence. Monocytes were cultured in RPMI-1640 medium supplemented with $10 \%$ fetal bovine serum (FBS), $1 \%$ L-glutamine, $1 \%$ penicillin/streptomycin and $100 \mathrm{ng} \mathrm{ml}^{-1} \mathrm{M}$-CSF (Peprotech) for 7 days to generate resting macrophages. T and $\mathrm{B}$ cells were obtained from peripheral blood of healthy donors using RosetteSep Human T Cell Enrichment Cocktail and RosetteSep Human B Cell Enrichment Cocktail (Stem Cell Technology), following the manufacturer's instructions. Neutrophils were enriched from Ficoll-isolated granulocytes, using an EasySep Human Neutrophil Enrichment Kit (StemCell Technologies), according to the manufacturer's instructions.

To analyse pluripotent haematopoietic stem cells and NK cell precursors, human bone marrow mononuclear cells were collected from Humanitas Biobank, upon approval by the Humanitas Research Hospital Ethical Committee (authorization 1516, issued on 26 February 2016). Frozen samples were thawed and vitality was assessed by trypan blue and Aqua LIVE/Dead- $405 \mathrm{~nm}$ staining (Invitrogen), before flow cytometry analysis.

Informed consent was obtained from all participants.

Fluorescence-activated cell sorting (FACS) analysis. Single-cell suspensions of bone marrow, blood, spleen, lung and liver were obtained and stained. A representative NK cell gating strategy is reported in Supplementary Fig. 1. A Foxp3/ Transcription Factor Staining Buffer Set (eBioscience) was used for intracellular staining of granzyme B and perforin. Cytofix/Cytoperm (BD Biosciences) was used for intracellular staining of IFN $\gamma$. Liver ILC1 were identified as NK1. $1^{+} \mathrm{CD}^{-}{ }^{-} \mathrm{CD} 49 \mathrm{a}^{+} \mathrm{CD} 49 \mathrm{~b}^{-}$cells. Formalin $4 \%$ and methanol $100 \%$ were used for intracellular staining of IRAK4, pIRAK4, pS6 and JNK. The following mouse antibodies were used: CD45-BV605, -BV650 or -PerCp-Cy5.5 (clone 30-F11); CD45.1-BV650 (clone A20); CD45.2-APC, -BV421 (clone 104); CD3ePerCP-Cy5.5 or -APC (clone 145-2C11); CD19-PerCP-Cy5.5, -eFluor450 (clone 1D3); NK1.1-PE, -APC, -eFluor450 or -Biotin (clone PK136); CD11b-BV421, -BV450, -BV785 (clone M1/70); CD27-FITC or -APC-eFluor780 (clone LG.7F9); CD4-FITC (clone RM 4-5); CD8-PE (clone 53-6.7); KLRG-1-BV421 (clone 2F1); NKG2D-APC (clone CX5); DNAM-1-APC (clone 10E5); Ly49H-PECF594 (clone 3D10); Granzyme B-PE (clone NGZB); Perforin-PE (clone eBioOMAK-D); IFN -Alexa700 or -APC (clone XMG1.2); CD107a-Alexa647 (clone 1D4B); FasL-APC (clone MFL3); Lineage Cell Detection Cocktail-Biotin; Sca-1-FITC (clone D7); CD117-PE or -Biotin (clone 3C11); CD127-eFluor450 (clone A7R34); CD135APC or -Biotin (clone A2F10.1); CD244-PE (clone 2B4); CD122-PE-CF594 (clone TM-Beta1); CD49b-PE-Cy7 or Biotin (clone DX5), CD49a-APC (clone Ha31/8), from BD Bioscience, eBioscience, BioLegend or Miltenyi Biotec. The following human antibodies were used: CD56-PE (clone CMSSB); CD3-FITC (clone UCHT1); CD16-Pacific Blue (clone 3G8); CD34-PE-Vio770 (clone AC136); CD117-BV605 (clone 104D2); NKp46-BV786 (clone 9E2/NKp46); CD45-PerCP (clone 2D1); CD19-APC-H7 (clone SJ25C1); CD14-APC-H7 (clone M5E2); CD66b-APC-Vio770 (clone REA306), from BD Bioscience, eBioscience or Miltenyi Biotec. Biotinylated anti-hSIGIRR (R\&D Systems) and streptavidin Alexa Fluor 647 (Invitrogen) were used to stain IL-1R8 in human cells. Human NKT cells were detected using PE-CD1d tetramers loaded with $\alpha$ GalCer (ProImmune, Oxford, UK). Antibodies to detect protein phosphorylation were as follows: p-IRAK4 Thr345/Ser346 (clone D6D7), IRAK4, p-S6-Alexa647 Ser235/236 (clone D57.2.2E); p-SAPK/JNK Thr183/Tyr185 (clone 81E11), from Cell Signaling Technology. A goat anti-rabbit Alexa Fluor 647 secondary antibody (Invitrogen) was used to stain p-IRAK4, IRAK4 and p-SAPK/JNK. Results are reported as mean fluorescence intensity normalized on isotype control or fluorescence minus one. Cell viability was determined by Aqua LIVE/Dead-405 nm staining (Invitrogen) or Fixable Viability Dye (FVD) eFluor 780 (eBioscience); negative cells were considered viable. Cells were analysed on an LSR Fortessa or FACSVerse (BD Bioscience). Data were analysed with FlowJo software (Treestar) Quantitative PCR. Total RNA was extracted using Trizol reagent (Invitrogen) following the manufacturer's recommendations. RNA was further purified using an miRNeasy RNA isolation kit (Qiagen) or Direct-zol RNA MiniPrep Plus (Zymo Research). cDNA was synthesized by reverse transcription using a High Capacity cDNA Archive Kit (Applied Biosystems) and quantitative real-time PCR was performed using SybrGreen PCR Master Mix (Applied Biosystems) in a CFX96 Touch Real-Time PCR Detection System (Bio-Rad). PCR reactions were performed with $10 \mathrm{ng}$ of DNA. Data were analysed with the $2^{(-\Delta \mathrm{CT})}$ method. Data were normalized on the basis of GAPDH, $\beta$-actin or $18 \mathrm{~S}$ expression, as indicated determined in the same sample. Analysis of all samples was performed in duplicate. Primers were designed according to the published sequences and listed as follows: s18/S18: forward 5'-ACT TTC GAT GGT AGT CGC CGT-3', reverse 5'-CCT TGG ATG TGG TAG CCG TTT-3'; Gapdh/GAPDH: forward 5'-GCA AAG TGG AGA TTG TTG CCA T- $3^{\prime}$, reverse $5^{\prime}$-CCT TGA CTG TGC CGT TGA ATT T- $3^{\prime}$; Actb/ ACTB: forward 5'-CCC AAG GCC AAC CGC GAG AAG AT-3', reverse 5'-GTC CCG GCC AGC CAG GTC CAG-3'; Illr8: forward 5'-AGA GGT CCC AGA AGA GCC AT-3', reverse 5'-AAG CAA CTT CTC TGC CAA GG-3'; IL1R8: forward 5'-ATG TCA AGT GCC GTC TCA ACG- ${ }^{\prime}$, reverse 5'-GCT GCG GCT TTA GGA TGA AGT-3'; Illrl: forward $5^{\prime}$-TGC TGT CGC TGG AGA TTG AC-3', reverse $5^{\prime}$-TGG AGT AAG AGG ACA CTT GCG AA- $3^{\prime}$; Ill $r 2$ : forward 5'-AGT GTG CCC TGA CCT GAA AGA-3' ${ }^{\prime}$, reverse $5^{\prime}$-TCC AAG AGT ATG GCG CCC T-3'; Illr3: forward 5'-GGC TGG CCC GAT AAG GAT- ${ }^{\prime}$, reverse 5'-GTC CCC AGT CAT CAC AGC G-3'; Illr4: forward 5'-GAA TGG GAC TTT GGG CTT TG-3', reverse 5'-GAC CCC AGG ACG ATT TAC TGC-3'; Illr5: forward 5'-GCT CGC CCA GAG TCA CTT TT-3', reverse 5'-GCG ACG ATC ATT TCC GAC TT-3'; Illr6: forward 5'-GCT TTT CGT GGC AGC AGA TAC-3', reverse 5'-CAG ATT TAC TGC CCC GTT TGT T-3'; 16S: forward 5'-AGA GTT TGA TCC TGG CTC AG- ${ }^{\prime}$, reverse $5^{\prime}$-GGC TGC TGG CAC GTA GTT AG-3'.

Purification of mouse leukocytes. Splenic NK cells and bone marrow neutrophils were enriched by MACS according to the manufacturer's instructions (Miltenyi Biotec). Purity of NK cells was about $90 \%$ as determined by FACS. The purity of neutrophils was $\geq 97.5 \%$. NK cells were stained (CD45-BV650, NK1.1-PE, CD3e-APC, CD11b-BV421, CD27-FITC) and sorted on a FACSAria cell sorter (BD Bioscience) to obtain high-purity NK cells and NK cell populations $\left(\mathrm{CD} 11 \mathrm{~b}^{\text {low }} \mathrm{CD} 27^{\text {low }}, \mathrm{CD} 11 \mathrm{~b}^{\text {low }} \mathrm{CD} 27^{\text {high }}, \mathrm{CD} 11 \mathrm{~b}^{\text {high }} \mathrm{CD} 27^{\text {high }}\right.$ and $\left.\mathrm{CD} 11 \mathrm{~b}^{\text {high }} \mathrm{CD} 27^{\mathrm{low}}\right)$. Splenic B and T lymphocytes were stained (CD45-PerCP, CD3e-APC, CD4-FITC, CD8-PE, CD19-eFluor450) and sorted. The purity of each population was $\geq 98 \%$. Resulting cells were processed for mRNA extraction or used for adoptive transfer or co-culture experiments. In vitro-derived macrophages were obtained from bone marrow total cells. Bone marrow cells were cultured in RPMI-1640 medium supplemented with 10\% FBS, 1\% L-glutamine, $1 \%$ penicillin/ streptomycin and $100 \mathrm{ng} \mathrm{ml}^{-1} \mathrm{M}$-CSF (Peprotech) for 7 days to generate resting macrophages. Bone marrow cells were cultured in RPMI-1640 medium supplemented with $10 \% \mathrm{FBS}, 1 \% \mathrm{~L}$-glutamine, $1 \%$ penicillin/streptomycin and $20 \mathrm{ng} \mathrm{ml}^{-1}$ GM-CSF (Peprotech) for 7 days to generate dendritic cells.

Confocal microscopy. Mouse splenic NK cells were enriched by magnetic cell sorting, left to adhere on poly-D-lysine (Sigma-Aldrich) coated coverslips, fixed with $4 \%$ PFA, permeabilized with $0.1 \%$ Triton X-100 and incubated with blocking buffer (5\% normal donkey serum (Sigma-Aldrich), 2\% BSA, 0.05\% Tween). Cells were then stained with biotin-conjugated goat polyclonal anti-SIGIRR antibody or biotin-conjugated normal goat IgG as control (both R\&D Systems) $\left(10 \mu \mathrm{g} \mathrm{ml}^{-1}\right)$ followed by Alexa Fluor 488-conjugated donkey anti-goat IgG antibody (Molecular Probes) and 4',6-diamidino-2-phenylindole (DAPI) (Invitrogen). Coverslips were mounted with the antifade medium FluorPreserve Reagent (EMD Millipore) and analysed with an Olympus Fluoview FV1000 laser scanning confocal microscope with a $40 \times$ oil immersion lens (numerical aperture 1.3).

STED microscopy. Human NK cells were enriched and left to adhere on polyD-lysine (Sigma-Aldrich) -coated coverslips, stimulated with IL-18 (50 $\mathrm{ng} \mathrm{ml}^{-1}$, $1 \mathrm{~min}, 5 \mathrm{~min}, 10 \mathrm{~min}$ ), fixed with $4 \%$ PFA, incubated with $5 \%$ normal donkey serum 
(Sigma-Aldrich), 2\% BSA, 0.05\% Tween in PBS2+ (pH 7.4) (blocking buffer), and then with biotin-conjugated goat polyclonal anti-human IL-1R8 antibody or biotin-conjugated normal goat IgG (all from R\&D Systems) and mouse monoclonal anti-IL-18R $\alpha$ (Clone 70625; R\&D Systems) or mouse IgG1 (Invitrogen), all diluted at $5 \mu \mathrm{g} \mathrm{ml}^{-1}$ in blocking buffer, followed by Alexa Fluor 488-conjugated donkey anti-goat IgG antibody and Alexa Fluor 555 donkey anti-mouse IgG antibody (both from Molecular Probes). Mowiol was used as mounting medium. STED xyz images were acquired in a unidirectional mode with a Leica SP8 STED3X confocal microscope system. Alexa Fluor 488 was excited with a $488 \mathrm{~nm}$ argon laser and emission collected from 505 to $550 \mathrm{~nm}$ applying a gating between 0.4 and $7 \mathrm{~ns}$ to avoid collection of reflection and autofluorescence. Alexa Fluor 555 was excited with a $555 / 547 \mathrm{~nm}$-tuned white light laser and emission collected from 580 to $620 \mathrm{~nm}$. Line sequential acquisition was applied to avoid fluorescence overlap. The $660 \mathrm{~nm}$ CW-depletion laser ( $80 \%$ of power) was used for both excitations. Images were acquired with Leica HC PL APO $100 \times / 1.40$ numerical aperture oil STED White objective at 572.3 milli absorption unit (mAU). CW-STED and gated CW-STED were applied to Alexa Fluor 488 and Alexa Fluor 555, respectively. Collected images were de-convolved with Huygens Professional software.

3'-mRNA sequencing and analysis. Splenic NK cells (from six mice per genotype and pooled in pairs) were purified as described above and stimulated with IL-18 (MBL) (20 $\mathrm{ng} \mathrm{ml}^{-1}$ for $4 \mathrm{~h}$ ). RNA was prepared as described above. A QuantSeq 3'mRNA-seq Library Prep Kit for Illumina (Lexogen) was used to generate libraries, which were sequenced on the NextSeq (Illumina; 75 bp PE). The fastq sequence files were assessed using the fastqc program. The reads were first trimmed using bbduk in the bbmap suite of software ${ }^{32}$ to remove the first 12 bases and a contaminant kmer discovery length of 13 was used for contaminant removal. Regions of length 20 or above with average quality of less than 10 were trimmed from the end of the read. The reads were then trimmed to remove trailing polyG and polyA runs using cutadapt ${ }^{33}$ and the quality of the remaining reads reassessed with fastqc. The trimmed reads were aligned to the $\mathrm{mm} 10$ genomic reference and reads assigned to features in the $\mathrm{mm} 10$ annotation using the STAR program ${ }^{34}$. Differential expression analysis used the generalized linear model functions in the $\mathrm{R} /$ bioconductor $^{35}$ edgeR package ${ }^{36}$ with TMM normalization. Gene set analysis used the romer ${ }^{37}$ function in the $\mathrm{R} /$ bioconductor package limma ${ }^{38}$. Metascape (http://metascape.org) was used to enrich genes for Gene Ontology biological processes, KEGG Pathway and Reactome Gene Sets.

Measurement of cytokines. A BD Cytometric Bead Array (CBA) mouse inflammation kit (BD) or Duoset ELISA kits (R\&D Systems) were used to measure cytokines. In vitro functional assays. Total mouse splenocytes or enriched mouse or human NK cells were cultured in RPMI-1640 medium supplemented with 10\% FBS $1 \%$ L-glutamine, $1 \%$ penicillin/streptomycin and treated with IL-2, IL-12, IL-15 (Peprotech), IL-18 (MBL), IL-1 $\beta$ (Peprotech) and PMA-Ionomycin (SigmaAldrich), as specified. FasL expression was evaluated upon treatment for $45 \mathrm{~min}$ with IL-18 (50 $\left.\mathrm{ng} \mathrm{ml}^{-1}\right)$, IL-15 (50 $\left.\mathrm{ng} \mathrm{ml}^{-1}\right)$, IL-2 $\left(20 \mathrm{ng} \mathrm{ml}^{-1}\right)$ and IL-12 (10 $\left.\mathrm{ng} \mathrm{ml}^{-1}\right)$. IFN $\gamma$ production was analysed upon $16 \mathrm{~h}$ of treatment with IL-12 $\left(20 \mathrm{ng} \mathrm{ml}^{-1}\right)$ and IL-18 $\left(20 \mathrm{ng} \mathrm{ml}^{-1}\right)$ or IL-1 $\beta\left(20 \mathrm{ng} \mathrm{ml}^{-1}\right)$, by intracellular staining using a BD Cytofix/Cytoperm Fixation/Permeabilization Kit, following the manufacturer's instructions, or by ELISA. Granzyme B and perforin intracellular staining was performed upon $18 \mathrm{~h}$ of stimulation with IL-12 $\left(10 \mathrm{ng} \mathrm{ml}^{-1}\right)$, IL-15 $\left(10 \mathrm{ng} \mathrm{ml}^{-1}\right)$ and IL-18 (50 $\left.\mathrm{ng} \mathrm{ml}^{-1-1}\right)$, using a Foxp3/Transcription Factor Staining Buffer Set (eBioscience). CD107a-Alexa Fluor 647 antibody was added during the $4 \mathrm{~h}$ culture and analysed by flow cytometry. BD GolgiPlug (containing Brefeldin) and BD GolgiStop (containing Monensin) were added $4 \mathrm{~h}$ before intracellular staining. PMA ( $50 \mathrm{ng} \mathrm{ml}^{-1}$ ) and ionomycin $\left(1 \mu \mathrm{g} \mathrm{ml}^{-1}\right)$ were added $4 \mathrm{~h}$ before intracellular staining, when specified.

NK-dendritic-cell co-culture experiments were performed as previously described $^{39}$. Dendritic cells were treated with LPS from Escherichia coli O55:B5 (Sigma-Aldrich; $1 \mu \mathrm{g} \mathrm{ml}^{-1}$ ) or CpG ODN 1826 (Invivogen; $3 \mu \mathrm{g} \mathrm{ml}^{-1}$ ) and with anti-mIL-18 neutralizing antibody (BioXCell, Clone YIGIF74-1G7; $5 \mu \mathrm{g} \mathrm{ml}^{-1}$ ) or Rat Isotype Control (BioXCell, Clone 2A3).

IFN $\gamma$ and CD107a expression upon viral infection was analysed by flow cytometry upon $4 \mathrm{~h}$ treatment with BD GolgiPlug, BD GolgiStop and IL-2 $\left(500 \mathrm{U} \mathrm{ml}^{-1}\right)$.

Phosphorylation of IRAK4, S6 and JNK was analysed upon 15-30 min stimulation with IL-18 $\left(10 \mathrm{ng} \mathrm{ml}^{-1}\right)$.

Human primary NK cell transfection. Human NK cells were enriched from peripheral blood of healthy donors and transfected with Dharmacon Acell siRNA (GE Healthcare) using Accell delivery medium (GE Healthcare), following the manufacturer's instructions. SIGIRR-specific siRNA $(1 \mu \mathrm{M})$ (On-Target Plus; Dharmacon, GE Healthcare) comprised $250 \mathrm{nM}$ of the four following antisense sequences: I, AGU UUC GCG AGC CGA GAU CUU; II, UAC CAG AGC AGC ACG UUG AUU; III, UGA CCC AGG AGU ACU CGU GUU; IV, CUU CCC GUC GUU UAU CUC CUU (all $5^{\prime}$ to $3^{\prime}$ ).
Generation of bone marrow chimaeras. $I l 1 r 8^{-1-}$ and $I l 1 r 8^{+/+}$mice were lethally irradiated with a total dose of $900 \mathrm{cGy}$. Two hours later, mice were injected in the retro-orbital plexus with $4 \times 10^{6}$ nucleated bone marrow cells obtained by flushing of the cavity of freshly dissected femurs from wild-type or $111 \mathrm{r}^{-1-}$ donors. Competitive bone marrow chimaeric mice were generated by reconstituting recipient mice with 50\% CD45.1 $\mathrm{Illr} \mathrm{r}^{+/+}$and $50 \% \mathrm{CD} 45.2 \mathrm{Ill} 1 \mathrm{r} 8^{-/-}$bone marrow cells. Recipient mice received gentamycin $\left(0.8 \mathrm{mg} \mathrm{ml}^{-1}\right.$ in drinking water) starting 10 days before irradiation and for 2 weeks after irradiation. NK cells of chimaeric mice were analysed 8 weeks after bone marrow transplantation.

Depletion and blocking experiments. Mice were treated intraperitoneally with $200 \mu \mathrm{g}$ of specific mAbs (mouse anti-NK1.1, clone PK136; mouse isotype Control, clone C1.18.4; rat anti-mIL-18, clone YIGIF74-1G7; rat isotype Control, clone 2A3; rat anti-IFN $\gamma$, clone XMG1.2; rat IgG1 HRPN; mouse anti-IL-17A, clone 17F3 mouse isotype Control, clone MOPC-21; rat anti-CD4/CD8, clone GK1.5/YTS rat isotype Control, clone LTF-2 (all from BioXCell)) and then with $100 \mu \mathrm{g}$ once (anti-NK1.1) or three times (anti-IL-18, anti-IFN $\gamma$, anti-IL-17A, anti-CD4/CD8) a week for the entire duration of the experiment.

Microflora depletion. Six-week-old mice were treated every day for 5 weeks by oral gavage with a cocktail of antibiotics (ampicillin (Pfizer) $10 \mathrm{mg} \mathrm{ml}^{-1}$, vancomycin (PharmaTech Italia) $10 \mathrm{mg} \mathrm{ml}^{-1}$, metronidazol (Società Prodotti Antibiotici) $5 \mathrm{mg} \mathrm{ml}^{-1}$ and neomycin (Sigma-Aldrich) $10 \mathrm{mg} \mathrm{ml}^{-1}$ ). Control mice were treated with drinking water. A gavage volume of $10 \mathrm{ml} / \mathrm{kg}$ (body weight) was delivered with a stainless-steel tube without prior sedation of mice. DNA was isolated from bacterial faecal pellets with a PowerSoil DNA Isolation Kit (MO BIO Laboratories) and quantified by spectrophotometry at $260 \mathrm{~nm}$. PCR was performed with $10 \mathrm{ng}$ of DNA using SybrGreen PCR Master Mix (Applied Biosystems) in a CFX96 Touch Real-Time PCR Detection System (Bio-Rad). Data were analysed with the $2^{(-\Delta C T)}$ method (Applied Biosystems, Real-Time PCR Applications Guide).

Cancer models. Mice were injected intraperitoneally with $25 \mathrm{mg} / \mathrm{kg}$ (body weight) of diethylnitrosamine (Sigma) at 15 days of age. They were euthanized 6, 8, 10 or 12 months later, to analyse liver cancer. Liver cancer score was based on the number and volume of lesions ( 0 : no lesions; 1 : lesion number $<3$, or lesion dimension $<3 \mathrm{~mm}$; 2: lesion number $<5$, or lesion dimension $<5 \mathrm{~mm}$; 3 : lesion number $<10$, or lesion dimension $<10 \mathrm{~mm}$; 4 : lesion number $<15$, or lesion dimension $<10 \mathrm{~mm}$ 5: lesion number $>15$, or lesion dimension $>10 \mathrm{~mm}$ ). Lung metastasis experiments were performed injecting intramuscularly the 3-MCA-derived mycoplasma-free sarcoma cell line MN/MCA1 $\left(10^{5} \text { cells per mouse in } 100 \mu \mathrm{l} \mathrm{PBS}\right)^{40}$. Primary tumour growth was monitored twice a week, and lung metastases were assessed by in vivo imaging and by macroscopic counting at the time of being euthanized 25 days after injection. Liver metastases were generated by injecting intrasplenically $1.5 \times 10^{5}$ mycoplasma-free colon carcinoma cells (MC38) ${ }^{21}$. Mice were euthanized 12 days after injection and liver metastases were counted macroscopically. MC38 cells were received from ATCC just before use. MN/MCA1 cells were authenticated morphologically by microscopy in vitro and by histology ex vivo. Tumour size limi at which mice were euthanized was based on major diameter (not more than $2 \mathrm{~cm}$ ). Viral infections. Mice were injected intravenously with $5 \times 10^{5}$ plaque-forming units of the tissue-culture-grown virus in PBS. Bacterial artificial chromosomederived MCMV strain MW97.01 has been previously shown to be biologically equivalent to MCMV strain Smith (VR-1399) and is hereafter referred to as wildtype $\mathrm{MCMV}^{41}$. Mice were euthanized 1.5 and 4.5 days after infection and viral titre was assessed by plaque assay, as previously described ${ }^{42,43}$. Newborn mice were infected intraperitoneally with 2,000 plaque-forming units of the MCMV strain MW97.01 and euthanized at day 7 after infection. Viral titre was assessed by plaque assay, as previously described ${ }^{42,43}$

Adoptive transfer. One million $I l 1 r 8^{+/+}$or $I l 118^{-/-}$sorted NK cells were injected intravenously in wild-type adult mice $5 \mathrm{~h}$ before MN/MCA or MC38 injection, or intraperitoneally in newborn mice $48 \mathrm{~h}$ after MCMV injection. Adoptively transferred NK cell engraftment, proliferative capacity and functionality (IFNproduction and degranulation after ex vivo stimulation) were assessed 3 and 7 days after injection.

In vivo proliferation. In vivo proliferation was measured using a Click-iT Edu Flow Cytometry Assay Kit (Invitrogen). Edu was injected intraperitoneally $(0.5 \mathrm{mg}$ per mouse), mice were euthanized $24 \mathrm{~h}$ later and cells were stained following the manufacturer's instructions and analysed by flow cytometry.

Immunohistochemistry. Frozen liver tissues were cut at $8 \mathrm{~mm}$ and then fixed with $4 \%$ PFA. Endogenous peroxidases were blocked with $0.03 \% \mathrm{H}_{2} \mathrm{O}_{2}$ for $5 \mathrm{~min}$ and unspecific binding sites were blocked with PBS $+1 \%$ FBS for $1 \mathrm{~h}$. Tissues were stained with polyclonal goat anti-mouse NKp46/NCR1 (R\&D Systems) and a Goaton-Rodent HRP polymer kit (GHP516, Biocare Medical) was used as secondary antibody. Reactions were developed with 3,3'-diaminobenzidine (Biocare Medical) and then slides were counterstained with haematoxylin. Slides were mounted with eukitt (Sigma-Aldrich). Images at $20 \times$ magnification were analysed with cell^ $\mathrm{F}$ software (Olympus). 
In vivo imaging. After feeding with AIN-76A alfalfa-free diet (Mucedola, Italy) for 2 weeks to reduce fluorescence background, mice were intravenously injected with XenoLight RediJect 2-deoxyglucosone (PerkinElmer) and 24h later 2-deoxyglucosone fluorescence was measured using a Fluorescence Molecular Tomography system (FMT 2000, Perkin Elmer). Acquired images were subsequently analysed with TrueQuant 3.1 analysis software (Perkin Elmer).

Data availability. The data discussed in this publication have been deposited in the NCBI Gene Expression Omnibus (GEO) under accession number GSE105043, or from the corresponding author. Source Data for Figs 3, 4 and Extended Data Fig. 5 are provided.

Statistical analysis. For animal studies, sample size was defined on the basis of past experience on cancer and infection models, to detect differences of $20 \%$ or greater between the groups ( $10 \%$ significance level and $80 \%$ power). Values were expressed as mean \pm s.e.m. or median of biological replicates, as specified. Oneway ANOVA or a Kruskal-Wallis test were used to compare multiple groups. A two-sided unpaired Student's $t$-test was used to compare unmatched groups with Gaussian distribution and Welch's correction was applied in cases of significantly different variance. A Mann-Whitney $U$-test was used in cases of non-Gaussian distribution. A ROUT test was applied to exclude outliers. $P \leq 0.05$ was considered significant. Statistics were calculated with GraphPad Prism version 6, GraphPad Software.

Statistics and reproducibility. Figure 1a, $n=4$ (B cells), $n=5$ (NKT cells), $n=9$ (T cells), $n=10$ (NK cells) donors; Fig. $1 \mathrm{~b}, n=5$ donors; Fig. $1 \mathrm{c}, n=8$ (NK cells) or $n=4$ (T cells) or $n=3$ (other leukocytes) mice; Fig. $1 \mathrm{~d}, n=5$ mice. Figure $1 \mathrm{~b}$, Representative experiment out of six performed. Figure 1a, c, d, one experiment performed.

Figure 2a, b, $n=8$ or $n=7$ (spleen, Il1 $r 8^{+/+}$liver) or $n=6\left(\operatorname{Il} 1 r 8^{-l-}\right.$ liver) mice; Fig. 2c, $n=6$ mice; Fig. 2d, $n=9\left(I l 1 r 8^{+/+}\right)$or $n=6\left(\right.$ Ill $\left.r 8^{-/-}\right)$mice; Fig. 2e, $n=5$ mice; Fig. 2f, $n=6$ mice; Fig. 2g, $n=4$ mice; Fig. $2 \mathrm{~h}, n=5$ mice; Fig. $2 \mathrm{i}, n=10$ wells Fig. 2j, $n=4$ (IRAK4), $n=6$ or $n=5$ (S6 Illr $8^{-1-}$ ) or $n=7$ (JNK Ill $r 8^{-1-}$ ) mice; Fig. $2 \mathrm{k}, n=3$ mice; Fig. $2 \mathrm{l}, n=9$ healthy donors; Fig. $2 \mathrm{~m}, n=4$ healthy donors. Representative experiments out of three (Fig. 2a, b), five (Fig. 2c), two (Fig. 2d, j), four (Fig. 2e) performed. Fig. 2f-m, one experiment performed.

Figure 3a, $n=8,10,11,13,14$ mice; Fig. 3b, c, $n=6$ mice; Fig. 3d, $n=10,12,13$ mice; Fig. 3e, $n=10,11$ mice; Fig. 3f, $n=5$, 6, 7 mice; Fig. $3 g$, $n=9$, 10 mice; Fig. $3 \mathrm{~h}$, $n=5,6$ mice; Fig. 3 i, $n=9,10$ or 12 mice; Fig. 3j, $n=6$ mice. Representative experiments out of 6 (Fig. 3e), 3 (Fig. 3a), 2 (Fig. 3d, f, g, h, i). Fig. 3b, c, j, one experiment performed.

Figure 4a, b, $n=5$ mice; Fig. 4c, $n=6, n=9$ mice; Fig. 4d, $n=4$ mice. Fig. 4a, two experiments were performed. Fig. $4 \mathrm{~b}-\mathrm{d}$, one experiment was performed.

31. Garlanda, C. et al. Intestinal inflammation in mice deficient in Tir8, an inhibitory member of the IL-1 receptor family. Proc. Natl Acad. Sci. USA 101, 3522-3526 (2004).

32. Bushnell, B. Bbmap: a fast, accurate, splice-aware aligner (Ernest Orlando Lawrence Berkeley National Laboratory, 2014).

33. Martin, M. Cutadapt removes adapter sequences from high-throughput sequencing reads. EMBnet.journal 17, http://dx.doi.org/10.14806/ej.17.1.200 (2011).

34. Dobin, A. et al. STAR: ultrafast universal RNA-seq aligner. Bioinformatics $\mathbf{2 9}$, 15-21 (2013).

35. Gentleman, R. C. et al. Bioconductor: open software development for computational biology and bioinformatics. Genome Biol. 5, R80 (2004).

36. Robinson, M. D., McCarthy, D. J. \& Smyth, G. K. edgeR: a Bioconductor package for differential expression analysis of digital gene expression data. Bioinformatics 26, 139-140 (2010).

37. Majewski, I. J. et al. Opposing roles of polycomb repressive complexes in hematopoietic stem and progenitor cells. Blood 116, 731-739 (2010).

38. Ritchie, M. E. et al. limma powers differential expression analyses for RNA-sequencing and microarray studies. Nucleic Acids Res. 43, e47 (2015).

39. Mingozzi, F. et al. Prolonged contact with dendritic cells turns lymph node-resident NK cells into anti-tumor effectors. EMBO Mol. Med. 8, 1039-1051 (2016).

40. Giavazzi, R., Alessandri, G., Spreafico, F., Garattini, S. \& Mantovani, A. Metastasizing capacity of tumour cells from spontaneous metastases of transplanted murine tumours. Br. J. Cancer 42, 462-472 (1980).

41. Wagner, M., Jonjić, S., Koszinowski, U. H. \& Messerle, M. Systematic excision of vector sequences from the BAC-cloned herpesvirus genome during virus reconstitution. J. Virol. 73, 7056-7060 (1999).

42. Jonjić, S., Pavić, I., Lucin, P., Rukavina, D. \& Koszinowski, U. H. Efficacious control of cytomegalovirus infection after long-term depletion of $\mathrm{CD}^{+}$ T lymphocytes. J. Virol. 64, 5457-5464 (1990).

43. Reddehase, M. J. et al. Interstitial murine cytomegalovirus pneumonia after irradiation: characterization of cells that limit viral replication during established infection of the lungs. J. Virol. 55, 264-273 (1985). 


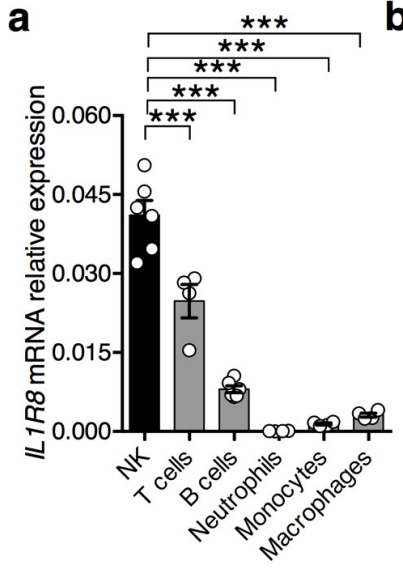

d
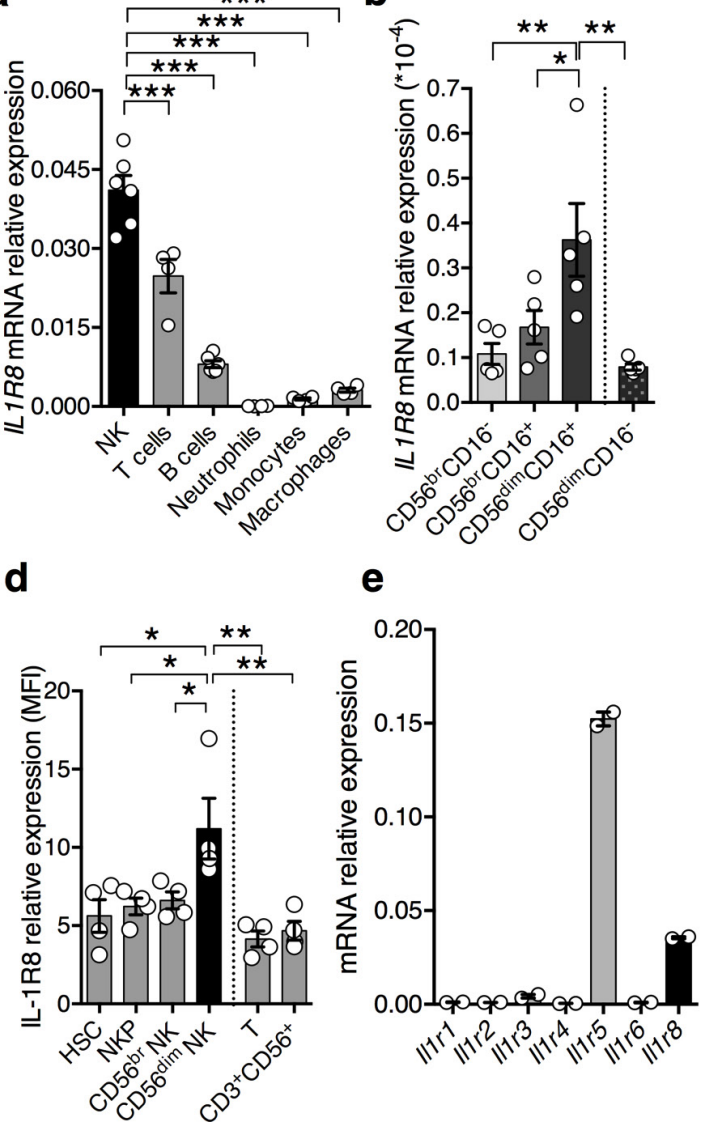

C

$f$

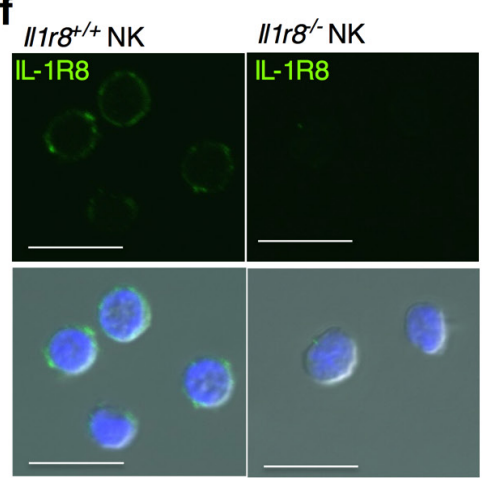

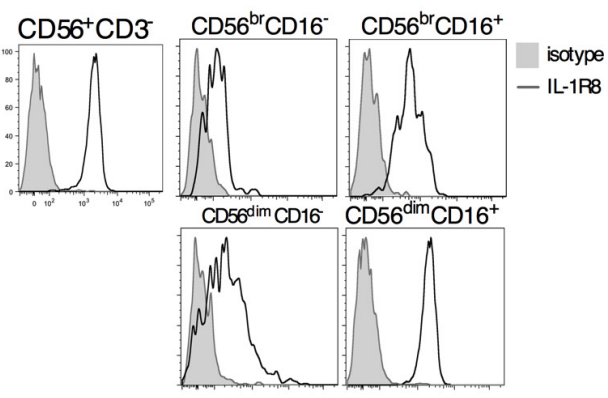

g

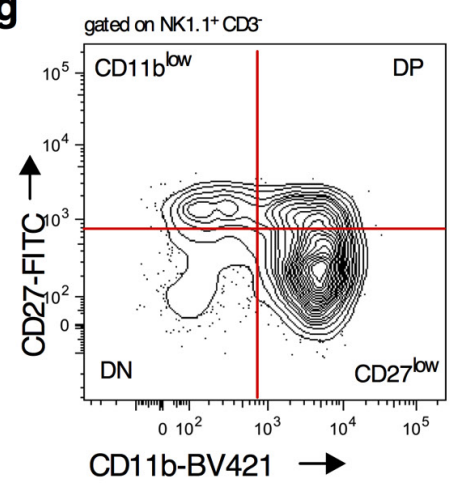

Extended Data Figure 1 | Expression of IL-1R8 in human and mouse NK cells. a, b, IL1R8 mRNA expression in human primary NK cells, compared with $\mathrm{T}$ and $\mathrm{B}$ cells, neutrophils, monocytes and in vitroderived macrophages (a) and in human primary NK cell maturation stages $\left(\mathrm{CD} 56^{\mathrm{br}} \mathrm{CD} 16^{-}, \mathrm{CD} 56^{\mathrm{br}} \mathrm{CD} 16^{+}, \mathrm{CD} 56^{\mathrm{dim}} \mathrm{CD} 16^{+}\right)$, and in the CD56 ${ }^{\mathrm{dim}} \mathrm{CD} 16^{-}$subset (b). c, Representative FACS plot of human NK cell subsets and histograms of IL-1R8 expression in NK cell subsets. d, IL-1R8 protein expression in human bone marrow precursors and mature cells. e, ILR family member (Illr1, Il1r2, Il1r3, Illr4, Il1r5, Il1r6, Il1r8) mRNA expression in mouse primary NK cells isolated from the spleen. f, IL-1R8 protein expression in mouse NK cells by confocal microscopy. Scale bars, $10 \mu \mathrm{m}$. g, Representative FACS plot of mouse NK cell subsets. a, b, d, $* P<0.05, * * P<0.01, * * * P<0.001$. One-way ANOVA. Mean \pm s.e.m. a, $n=6$ (NK and $\mathrm{B}$ cells) or $n=4$ donors; $\mathbf{b}, n=5$ donors; $\mathbf{d}, n=4$ donors; e, $n=2$ mice; $\mathbf{f}$, representative images out of four collected per group. a, b, d-f, One experiment performed. 
RESEARCH LETTER

a

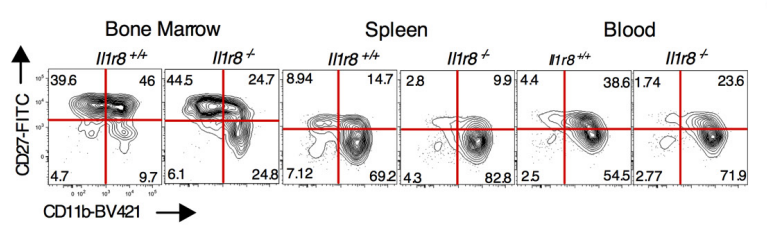

b

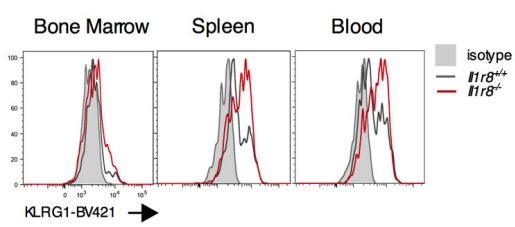

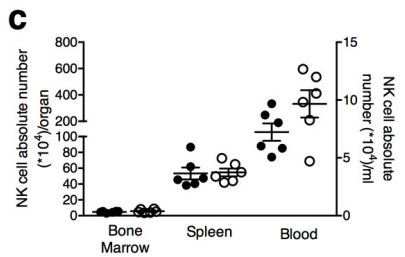
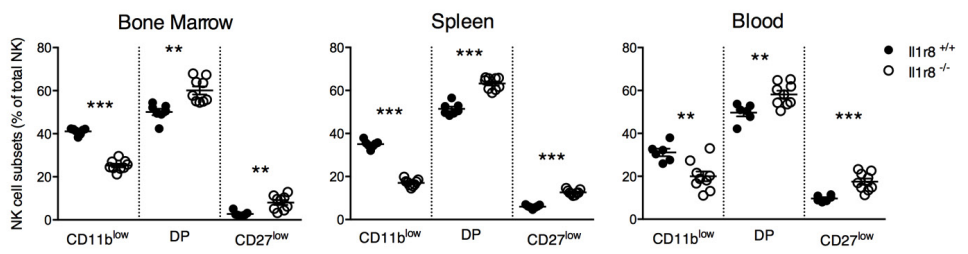

d
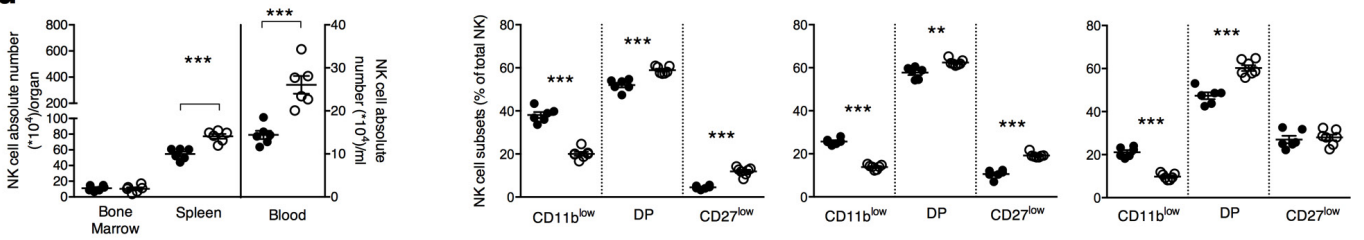

e
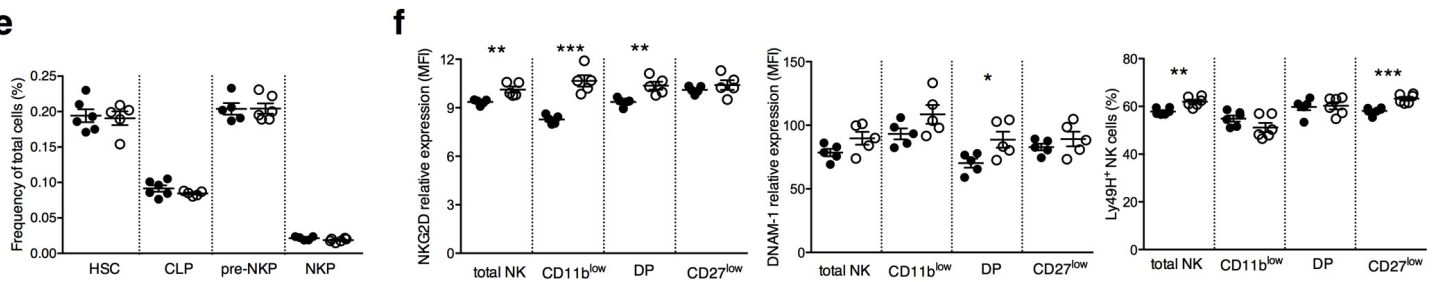

g

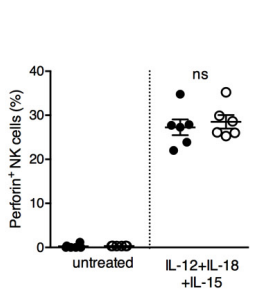

h
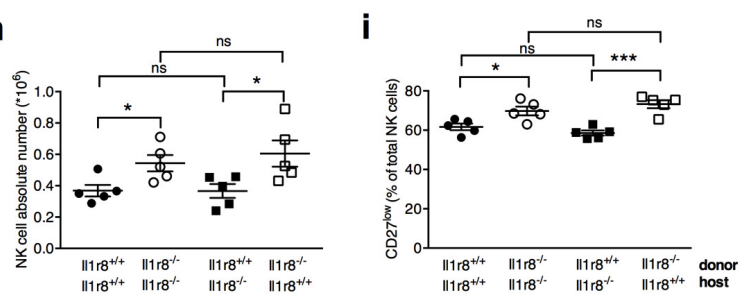

j

k

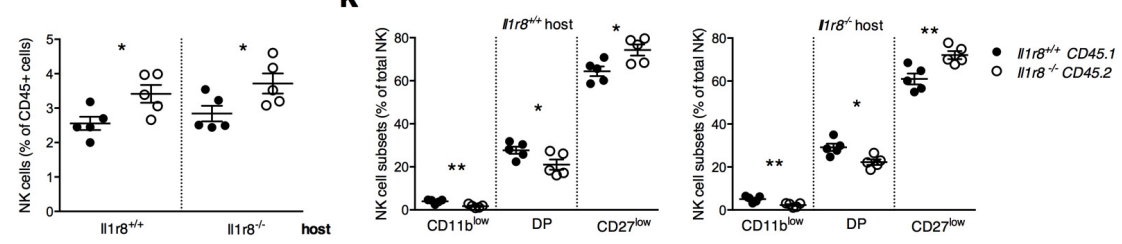

I

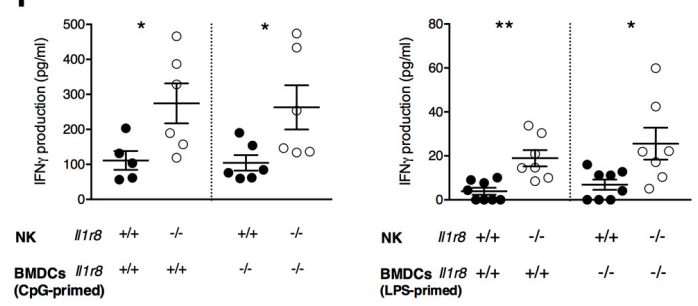

Extended Data Figure 2 | See next page for caption.

(c) 2017 Macmillan Publishers Limited, part of Springer Nature. All rights reserved. 
Extended Data Figure $2 \mid$ Phenotypic analysis of $111 r 8^{-1-}$ NK cells. a, b, Representative plot of fluorescence-activated cell sorting of mouse NK cell subsets in Ill $r 8^{+/+}$and $I l 1 r 8^{-/-}$mice (a) and histograms of KLRG1 expression in NK cells (b). c, d, NK absolute number and NK cell subsets (DN, CD11b $b^{\text {low }}$ DP and CD27 $7^{\text {low }}$ ) in bone marrow, spleen and blood of Ill $r 8^{+/+}$and Ill $r 8^{-1-}$ newborn mice at 2 (c) and 3 (d) weeks of age. e, Frequency of bone marrow precursors in $I l 1 r 8^{+/+}$and $I l 1 r 8^{-1-}$ mice. f, NKG2D, DNAM-1 and LY49H expression in peripheral NK cells and NK cell subsets of $I l 1 r 8^{+/+}$and $I l 1 r 8^{-1-}$ mice. g, Frequency of splenic Perforin ${ }^{+}$NK cell subsets upon stimulation in $I l 1 r 8^{+/+}$and $I l l r 8^{-1-}$ mice. $\mathbf{h}, \mathbf{i}$, Peripheral NK cell absolute number $(\mathbf{h})$ and CD27 $7^{\text {low }} \mathrm{NK}$ cell frequency (i) in bone marrow chimaeric mice upon reconstitution
(9 weeks). j, k, Peripheral NK cell (j) and NK cell subset (k) frequency in competitive chimaeric mice transplanted with $50 \%$ of $I l 1 r 8^{+/+}$ CD45.1 cells and $50 \%$ of $I l 1 r 8^{-1-}$ CD 45.2 cells upon reconstitution (9 weeks). Upon reconstitution, a defective engraftment (12\% instead of $50 \%$ engraftment) of $I l 1 \mathrm{r}^{-1-}$ stem cells was observed in competitive conditions. 1, IFN $\gamma$ production by $I l 1 r 8^{+/+}$and $I l 1 r 8^{-/-} \mathrm{NK}$ cells upon co-culture with LPS- or CpG-primed $I l 1 \mathrm{r}^{+/+}$and $I l 1 \mathrm{r}^{-1-}$ dendritic cells. c-l, $* P<0.05, * * P<0.01$, $* * * P<0.001$ between selected relevant comparisons, two-tailed unpaired Student's $t$-test. Centre values and error bars, mean \pm s.e.m. At least five animals per group were used. c, d, Three pooled experiments; $\mathbf{e}-\mathbf{l}$, one experiment was performed. 


\section{RESEARCH LETTER}
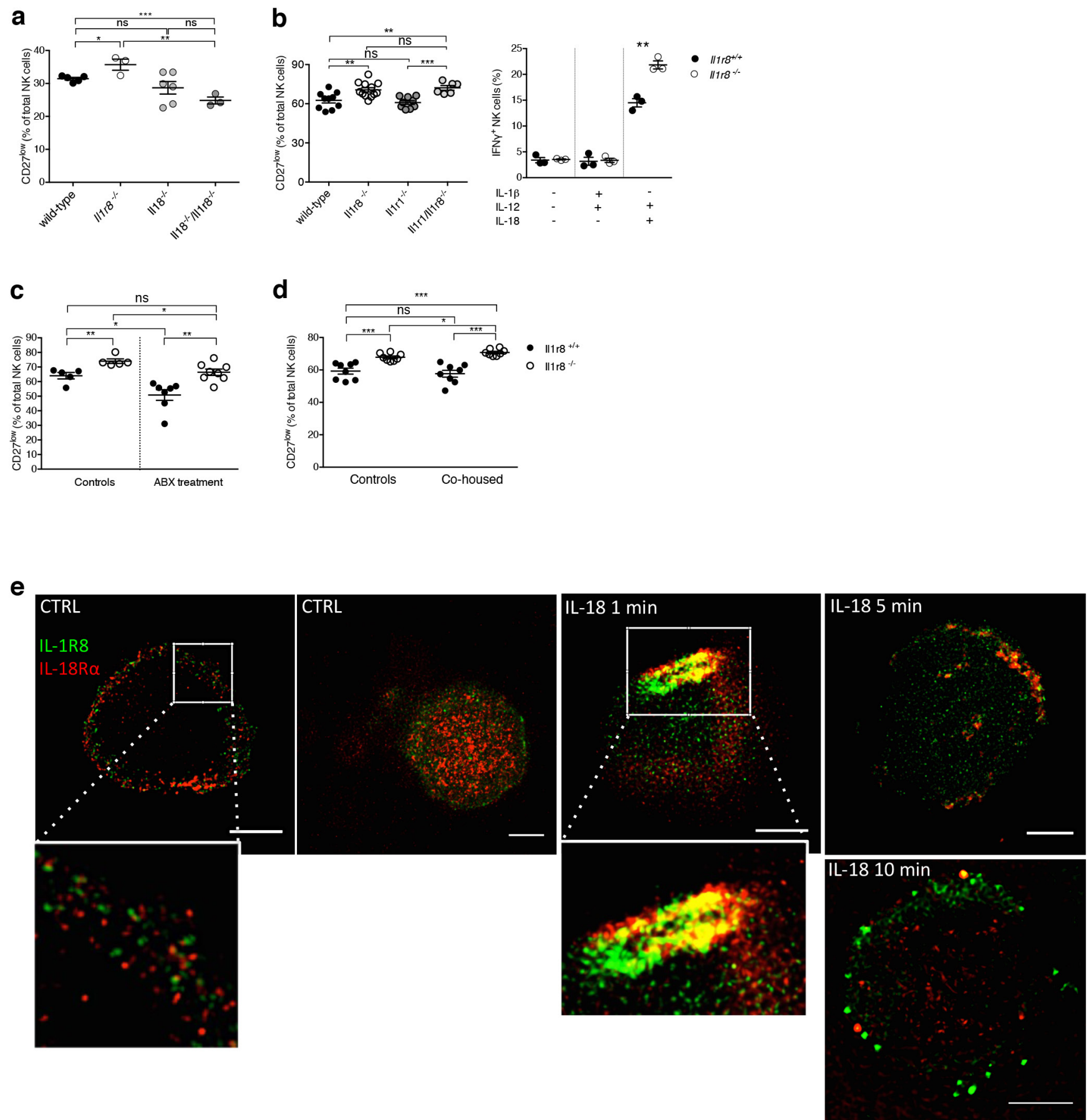

Extended Data Figure 3 Mechanism of IL-1R8-dependent regulation of NK cells. a, Splenic CD27 $7^{\text {low }} \mathrm{NK}$ cell frequency in wild-type, $\mathrm{Il} 1 \mathrm{r} \mathrm{8}^{-/-}$, $I l 18^{-/-}$and $I l 18^{-l-} / I l 1 r 8^{-l-}$ mice. b, Peripheral CD2 $27^{\text {low }}$ NK cell frequency in wild-type, Ill $r 8^{-1-}$, Ill $r 1^{-1-}$ and $I l 1 r 8^{-1-} I l 1 r 1^{-1-}$ mice (left) and IFN $\gamma$ production by splenic NK cells after IL-12 and IL-1 $\beta$ or IL-18 stimulation (right). c, d, Splenic CD2 $27^{\text {low }} \mathrm{NK}$ cell frequency in $\mathrm{Il} 1 \mathrm{r} 8^{+/+}$ and $I l 1 r 8^{-1-}$ mice upon commensal flora depletion (c) and breeding in co-housing conditions (d). e, STED microscopy of human NK cells stimulated with IL-18. Magnification bar, $2 \mu \mathrm{m}$. a-d, $* P<0.05$, $* * P<0.01$, $* * * P<0.001$ between selected relevant comparisons, two-tailed unpaired Student's $t$-test; Centre values and error bars, mean \pm s.e.m. a, $n=3$, 5 , or 6 mice; at least five animals per group were used (b-d). $\mathbf{a}-\mathbf{d}$, One experiment was performed. e, Representative images out of three collected from two donors. 


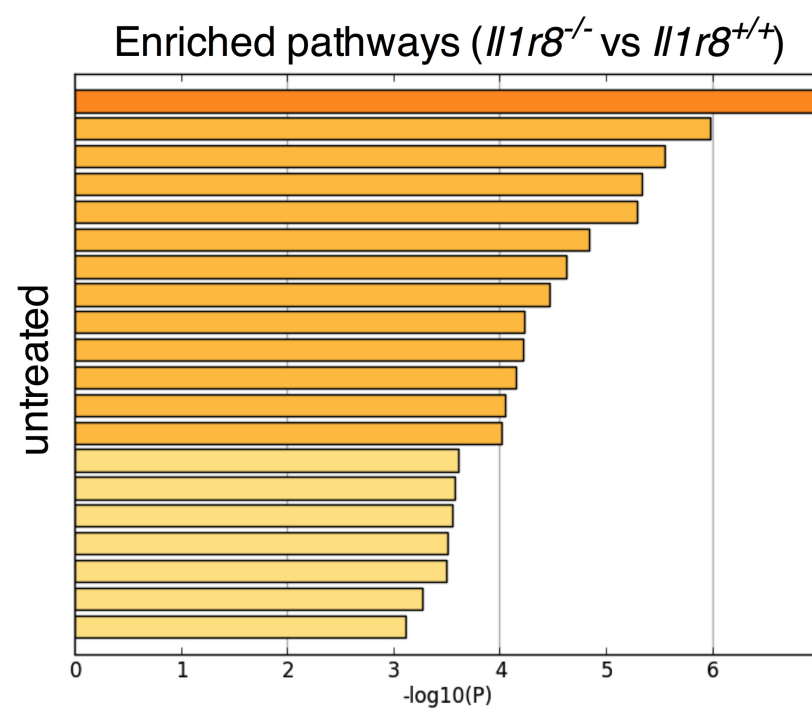

GO:0034097: response to cytokine

GO:0042110: $T$ cell activation

GO:0060548: negative regulation of cell death

ko04010: MAPK signaling pathway

GO:0071376: cellular response to corticotropin-releasing hormone stimulus

GO:0034968: histone lysine methylation

k004668: TNF signaling pathway

GO:0006396: RNA processing

GO:0022613: ribonucleoprotein complex biogenesis

GO:0001816: cytokine production

R-MMU-877312: Regulation of IFNG signaling

GO:0001890: placenta development

GO:0001704: formation of primary germ layer

GO:0030099: myeloid cell differentiation

GO:0000122: negative regulation of transcription from RNA polymerase II promoter

GO:1902532: negative regulation of intracellular signal transduction

R-MMU-352230: Amino acid transport across the plasma membrane

GO:0043604: amide biosynthetic process

GO:0010888: negative regulation of lipid storage

mmu05202: Transcriptional misregulation in cancer

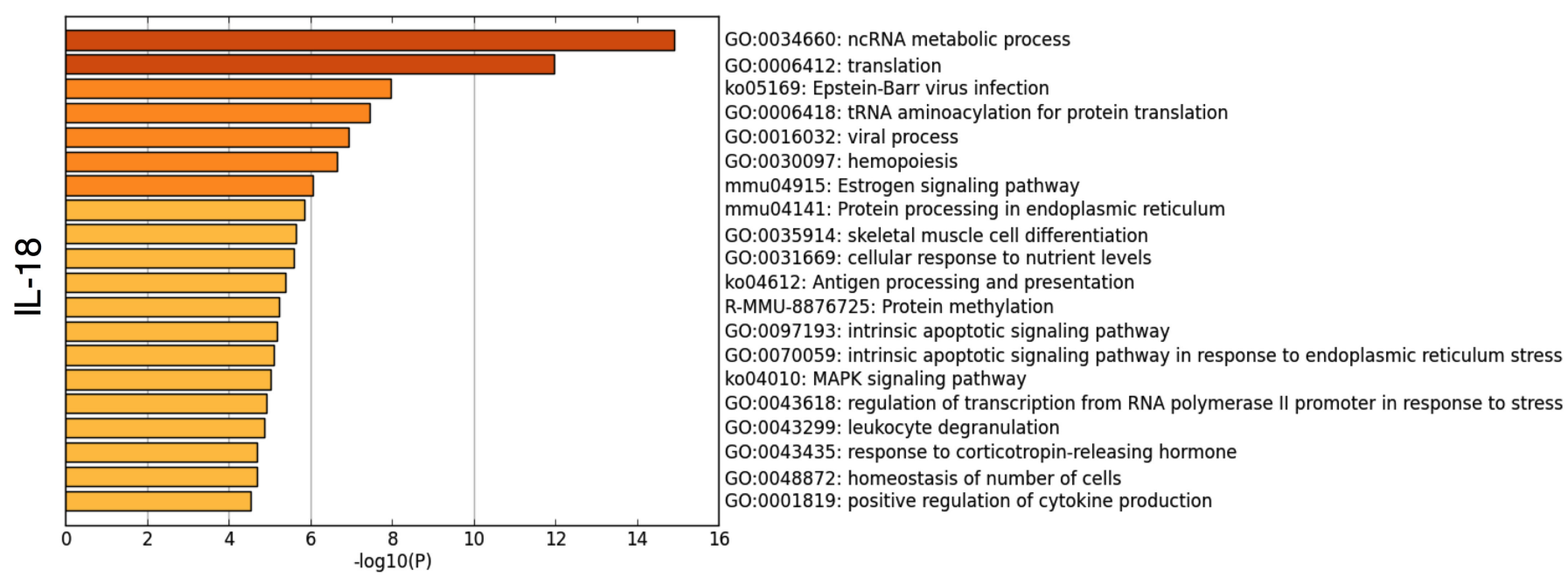

Extended Data Figure 4 | RNA-seq analysis of $I l 1 \mathrm{rB}^{+/+}$and $\mathrm{Ill} \mathrm{rr}^{-/-}$NK cells. Metascape analysis of enriched gene pathways of resting and IL-18activated $111 \mathrm{rr}^{+/+}$and $I l 11 \mathrm{r}^{-/-}$NK cells. See also Supplementary Table 1 and data deposited in the NCBI Gene Expression Omnibus under accession number GSE105043. 


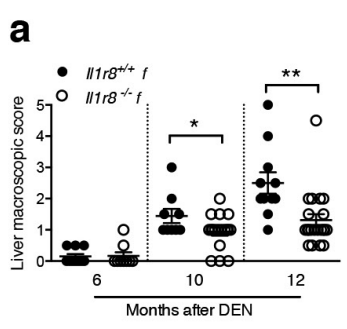

f

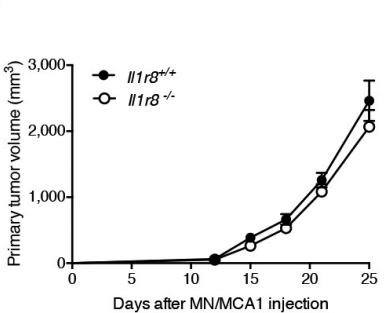

b

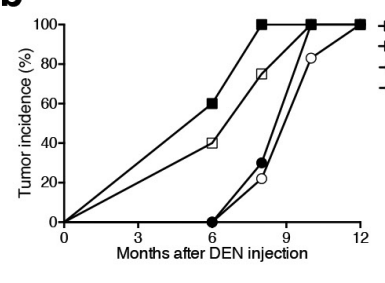

g

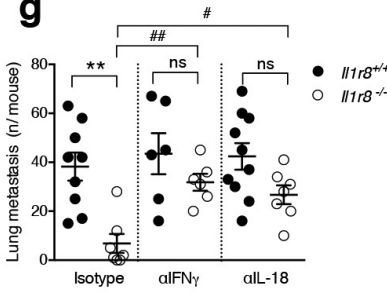

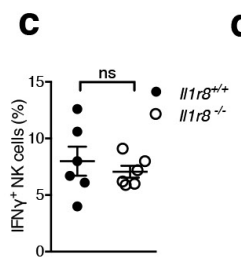

h

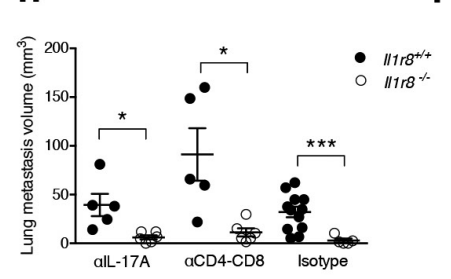

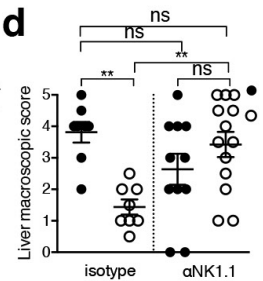

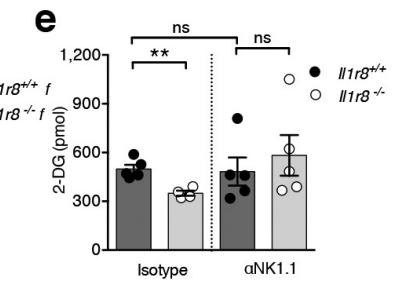

j

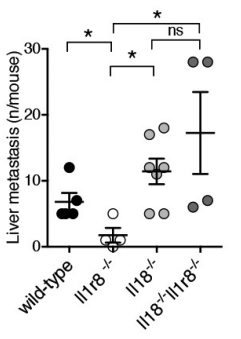

k

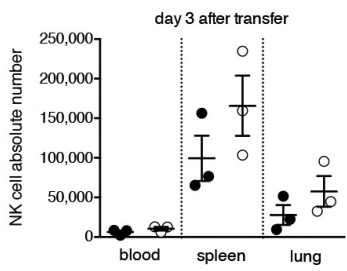

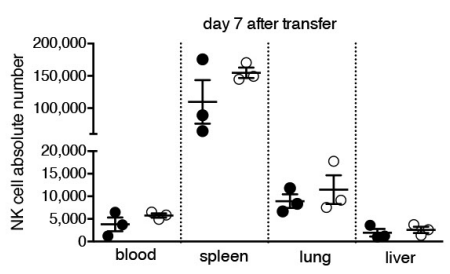

I

i

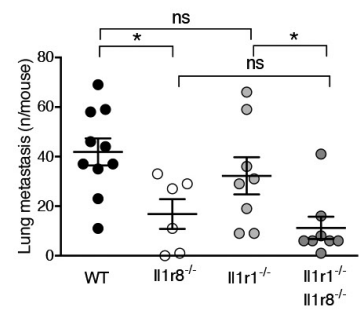

m

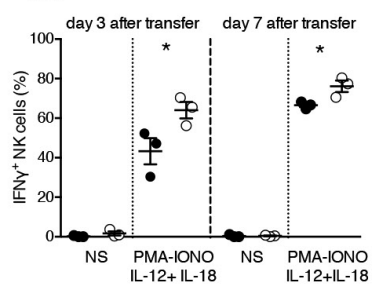

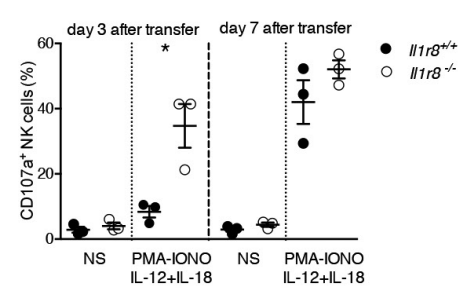

Extended Data Figure $5 \mid$ NK-cell-mediated resistance to hepatocellular carcinoma and metastasis in IL-1R8-deficient mice. a, Macroscopic score of liver lesions in female $I l 1 \mathrm{r}^{+/+}$and $I l 1 \mathrm{r}^{-/-}$mice 6, 10 and 12 months after diethylnitrosamine (DEN) injection. $\mathbf{b}$, Incidence of hepatocellular carcinoma in $I l 1 r 8^{+/+}$and $I l 1 r 8^{-1-}$ female and male mice. c, Frequency of IFN $\gamma^{+} \mathrm{NK}$ cells in spleen of $I l 1 r 8^{+/+}$and $I l 1 r 8^{-1-}$ tumourbearing mice. $\mathbf{d}$, Macroscopic score of liver lesions in female $\mathrm{Il} 1 \mathrm{r} \mathrm{r}^{+/+}$ and Ill $r 8^{-1-}$ mice upon NK cell depletion. e, 2-Deoxyglucosone (2-DG) quantification in lungs of $I l 1 r 8^{+/+}$and $I l 1 r 8^{-1-}$ tumour-bearing mice upon NK cell depletion. f, Primary tumour growth in $I l 1 r 8^{+/+}$and $I l 1 r 8^{-1-}$ mice (25 days after MN/MCA1 cell line injection). g, Number of lung metastases in $I l 1 r 8^{+/+}$and $I l 1 r 8^{-1-}$ MN/MCA1 sarcoma-bearing mice upon IFN $\gamma$ or IL-18 neutralization. h, Volume of lung metastases in $I l 1 \mathrm{r}^{+/+}$and $I l 1 \mathrm{r} 8^{-/-}$MN/MCA1-bearing mice upon depletion of IL-17A or $\mathrm{CD}^{+} / \mathrm{CD}^{+}$cells. i, Number of lung metastases in $\mathrm{Il1} 1 \mathrm{r}^{+/+}$and Ill $r 8^{-1-}$, Il1r1 $1^{-1-}$, Illr1 $1^{-1-} / I l 1 r 8^{-1-}$ MN/MCA1-bearing mice. $\mathbf{j}$, Number of liver metastases in Ill $r 8^{+/+}, I l 1 r 8^{-/-}, I l l 1^{-/-}, I l 18^{-/-} I l 1 r 8^{-/-}$MC38 $\mathbf{n}$
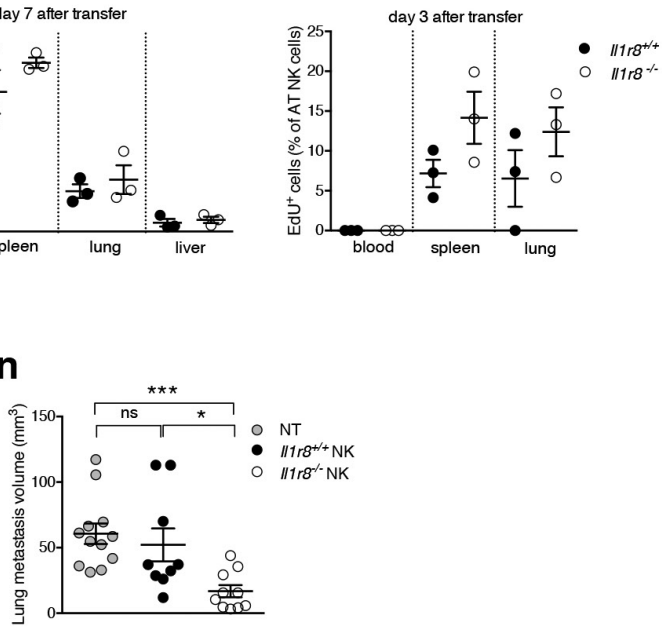

colon carcinoma-bearing mice. $\mathbf{k}, I l 1 r 8^{+/+}$and $I l 1 r 8^{-1-}$ NK cell absolute number 3 or 7 days after adoptive transfer. 1, In vivo Il $1 r 8^{+/+}$and Ill $r 8^{-1-}$ NK cell proliferation 3 days after adoptive transfer. $\mathbf{m}, E x$ vivo IFN $\gamma$ production and degranulation upon $4 \mathrm{~h}$ stimulation with PMA-ionomycin, IL-12 and IL-18 in adoptively transferred $I l 1 r 8^{+/+}$and $I l 1 r 8^{-/-} \mathrm{NK}$ cells. $\mathbf{n}$, Volume of lung metastases in $I l 1 r 8^{+/+}$MN/MCA1 sarcoma-bearing mice after adoptive transfer of $I l 1 r 8^{+/+}$and $I l 1 r 8^{-/-} \mathrm{NK}$ cells. a, $\mathbf{c}-\mathbf{e}, \mathbf{g}-\mathbf{j}, \mathbf{m}-\mathbf{n}, * P<0.05, * * P<0.01, * * * P<0.001$ between selected relevant comparisons, two-tailed unpaired Student's $t$-test or MannWhitney $U$-test. $\# P<0.05$, \#\#P<0.01, Kruskal-Wallis and Dunn's multiple comparison test. Centre values and error bars, mean \pm s.e.m. a, $n=9,10,11,18,21$ mice; $\mathbf{b}, n=8-21$ mice; c, $n=6$ mice; $\mathbf{d}, n=10$, 12,13 mice; e, $n=4$ (Illr $r 8^{-1-}$ isotype) or $n=5 ; \mathbf{f}, n=10 ; \mathbf{g}, n=6,7,9,10$ mice; $\mathbf{h}, n=5,6,12$ mice; $\mathbf{i}, n=6,8,10$ mice; $\mathbf{j}, n=4,5,7$ mice;

$\mathbf{k}, \mathbf{l}, \mathbf{m}, n=3$ mice; $\mathbf{n}, n=9,10,12$ mice. Representative experiment out of three (a, b), 2 (d), 6 (f), or one (c, e, g-n) experiments performed. NT, not treated. 

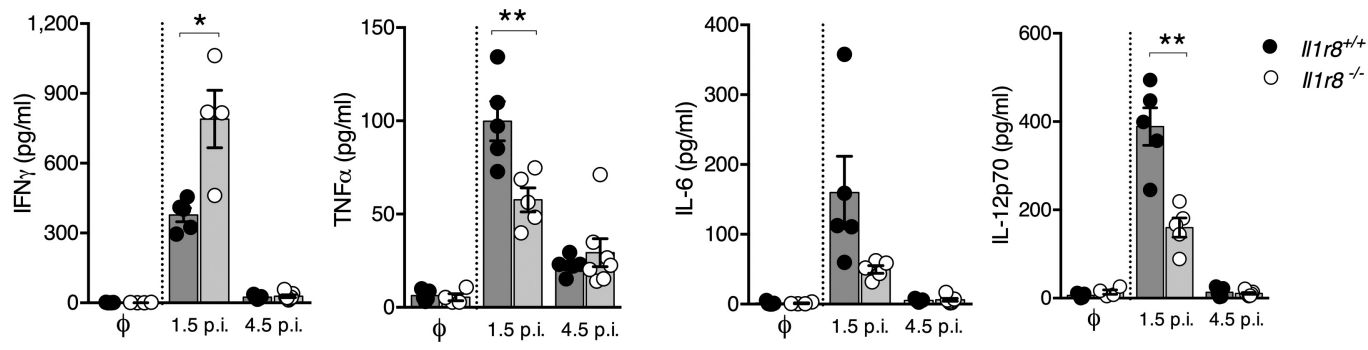

Extended Data Figure 6 | NK-cell-mediated anti-viral resistance in IL-1R8-deficient mice. Cytokine serum levels in $I l 1 r 8^{+/+}$and $I l 1 r 8^{-/-}$infected mice (1.5 and 4.5 days after infection). $* P<0.05, * * P<0.01, * * * P<0.001$, unpaired Student's $t$-test. Centre values and error bars, mean \pm s.e.m.; $n=5$ mice. One experiment was performed. 


\section{RESEARCH LETTER}

Extended Data Table 1 | Serum cytokine and liver enzyme levels in hepatocellular carcinoma-bearing mice

\begin{tabular}{|c|c|c|c|c|c|c|c|c|c|}
\hline $\begin{array}{c}\text { Cytokine } \\
\mathrm{pg} / \mathrm{mL}\end{array}$ & \multicolumn{3}{|c|}{6 months after DEN } & \multicolumn{3}{|c|}{ 8-10 months after DEN } & \multicolumn{3}{|c|}{12 months after DEN } \\
\hline IL-23 & $173.1 \pm 29.12$ & $247.3 \pm 15.16$ & 0.05 & $187.7 \pm 13.47$ & $343.4 \pm 66.29$ & 0.04 & $103.7 \pm 26.72$ & $138.6 \pm 37.51$ & 0.47 \\
\hline IL-17A & $69.98 \pm 9.88$ & $95.03 \pm 6.44$ & 0.07 & $56.41 \pm 7.46$ & $102.4 \pm 19.01$ & 0.04 & $38.13 \pm 10.39$ & $45.05 \pm 8.78$ & 0.62 \\
\hline IFN $\gamma$ & $295 \pm 72.78$ & $385.4 \pm 48.6$ & 0.32 & $357.5 \pm 57.63$ & $593.2 \pm 84.33$ & 0.05 & $195.4 \pm 65.29$ & $243.3 \pm 104$ & 0.72 \\
\hline IL- $1 \beta$ & $91.99 \pm 5.23$ & $58.68 \pm 7.29$ & 0.006 & $142.4 \pm 28.24$ & $60.35 \pm 4.42$ & 0.01 & $47.66 \pm 14.08$ & $29.81 \pm 7.66$ & 0.31 \\
\hline $\mathrm{TNF} \alpha$ & $163.5 \pm 7.16$ & $92.06 \pm 21.04$ & 0.01 & $194.6 \pm 28.03$ & $100.1 \pm 14.24$ & 0.008 & $94.77 \pm 14.24$ & $57.45 \pm 14.51$ & 0.13 \\
\hline CCL2 & $32.51 \pm 1.54$ & $24.1 \pm 5.64$ & 0.19 & $43.97 \pm 7.25$ & $25.42 \pm 1.37$ & 0.02 & $28.1 \pm 4.99$ & $19.72 \pm 1.23$ & 0.14 \\
\hline AST & $159.6 \pm 39.79$ & $101.0 \pm 1.87$ & 0.18 & $134.0 \pm 15.28^{* * *}$ & $97.0 \pm 8.0 * * *$ & 0.06 & $105.0 \pm 25.45$ & $89.0 \pm 5.1$ & 0.55 \\
\hline
\end{tabular}

*Samples with undetectable levels were not included in the analysis. ** Levels are $\mathrm{UI}^{-1}$

$* * * N=5,8$ months after diethylnitrosamine (DEN) injection. 


\section{Life Sciences Reporting Summary}

Nature Research wishes to improve the reproducibility of the work that we publish. This form is intended for publication with all accepted life science papers and provides structure for consistency and transparency in reporting. Every life science submission will use this form; some list items might not apply to an individual manuscript, but all fields must be completed for clarity.

For further information on the points included in this form, see Reporting Life Sciences Research. For further information on Nature Research policies, including our data availability policy, see Authors \& Referees and the Editorial Policy Checklist.

\section{- Experimental design}

\section{Sample size}

Describe how sample size was determined.

\section{Data exclusions}

Describe any data exclusions.

\section{Replication}

Describe whether the experimental findings were reliably reproduced.

\section{Randomization}

Describe how samples/organisms/participants were allocated into experimental groups.

5. Blinding

Describe whether the investigators were blinded to group allocation during data collection and/or analysis.
For animal studies, sample size was defined on the basis of past experience on cancer and infection models, in order to detect differences of $20 \%$ or grater between the groups (10\% significance level and $80 \%$ power).

ROUT test was applied to exclude outliers.

Most experiments were replicated several times with reproducible results as indicated in figure legend/Statistics and reproducibility.

Mice were randomized based on sex, age and weight.

In most in vivo experiments, the investigators were unaware of the genotype of the experimental groups.

Note: all studies involving animals and/or human research participants must disclose whether blinding and randomization were used.

6. Statistical parameters

For all figures and tables that use statistical methods, confirm that the following items are present in relevant figure legends (or in the Methods section if additional space is needed).

n/a $\mid$ Confirmed

$\bigotimes$ The exact sample size $(n)$ for each experimental group/condition, given as a discrete number and unit of measurement (animals, litters, cultures, etc.)

$\square$ A description of how samples were collected, noting whether measurements were taken from distinct samples or whether the same sample was measured repeatedly

$\bigotimes$ A statement indicating how many times each experiment was replicated

The statistical test(s) used and whether they are one- or two-sided (note: only common tests should be described solely by name; more complex techniques should be described in the Methods section)

$\bigotimes$ A description of any assumptions or corrections, such as an adjustment for multiple comparisons

$\triangle \square$ The test results (e.g. $P$ values) given as exact values whenever possible and with confidence intervals noted

$\square$ \ A clear description of statistics including central tendency (e.g. median, mean) and variation (e.g. standard deviation, interquartile range)

$\square$ \Clearly defined error bars 
Policy information about availability of computer code

\section{Software}

Describe the software used to analyze the data in this study.

For manuscripts utilizing custom algorithms or software that are central to the paper but not yet described in the published literature, software must be made available to editors and reviewers upon request. We strongly encourage code deposition in a community repository (e.g. GitHub). Nature Methods guidance for providing algorithms and software for publication provides further information on this topic.

\section{- Materials and reagents}

Policy information about availability of materials

8. Materials availability

Indicate whether there are restrictions on availability of unique materials or if these materials are only available for distribution by a for-profit company.

9. Antibodies

Describe the antibodies used and how they were validated for use in the system under study (i.e. assay and species).
IL-1R8-deficient mice are available upon a MTA signature.

The following murine antibodies were used: CD45-BV605, -BV650 or -PerCp-Cy5.5 (Clone 30-F11); CD45.1-BV650 (Clone A20); CD45.2-APC, -BV421 (Clone 104); CD3e-PerCP-Cy5.5 or -APC (Clone 145-2C11); CD19-PerCP-Cy5.5, -eFluor450 (Clone 1D3); NK1.1-PE, -APC, -eFluor450 or -Biotin (Clone PK136); CD11b-BV421, -BV450, -BV785 (Clone M1/70); CD27-FITC or -APC-eFluor780 (Clone LG.7F9); CD4-FITC (Clone RM 4-5); CD8-PE (Clone 53-6.7); KLRG-1-BV421 (Clone 2F1); NKG2D-APC (Clone CX5); DNAM-1-APC (Clone 10E5); Ly49H-PECF594 (Clone 3D10); Granzyme B-PE (Clone NGZB); Perforin-PE (Clone eBioOMAK-D); IFNy-Alexa700 or -APC (Clone XMG1.2); CD107a-Alexa647 (Clone 1D4B); FasL-APC (Clone MFL3); Lineage Cell Detection Cocktail-Biotin; Sca-1-FITC (Clone D7); CD117-PE or -Biotin (Clone 3C11); CD127-efluor450 (Clone A7R34); CD135-APC or -Biotin (Clone A2F10.1); CD244-PE (Clone 2B4); CD122-PE-CF594 (Clone TM-Beta1); CD49b-PE-Cy7 or Biotin (Clone DX5), CD49a-APC (Clone Ha31/8), from BD Bioscience, eBioscience, BioLegend or Miltenyi Biotec. Mouse anti-NK1.1, Clone PK136; Mouse Isotype Control, Clone C1.18.4; Rat anti-mIL-18, Clone YIGIF74-1G7; Rat Isotype Control, Clone 2A3; Rat anti-IFNy, Clone XMG1.2; Rat IgG1 HRPN; Mouse anti-IL-17A, Clone 17F3; Mouse Isotype Control, Clone MOPC-21; Rat anti-CD4/CD8, Clone GK1.5/YTS; Rat Isotype Control, Clone LTF-2.

The following human antibodies were used: CD56-PE (Clone CMSSB); CD3-FITC (Clone UCHT1); CD16-Pacific Blue (Clone 3G8); CD34-PE-Vio770 (Clone AC136); CD117-BV605 (Clone 104D2); NKp46-BV786 (Clone 9E2/NKp46); CD45-PerCP (Clone 2D1); CD19-APC-H7 (Clone SJ25C1); CD14-APC-H7 (Clone M5E2); CD66bAPC-Vio770 (Clone REA306), from BD Bioscience, eBioscience or Miltenyi Biotec. Biotinylated anti-hSIGIRR (R\&D Systems) and Streptavidin-Alexa647 (Invitrogen ${ }^{\mathrm{TM}}$ ) were used to stain IL-1R8 in human cells. Human NKT cells were detected using PECD1d tetramers loaded with aGalCer (Prolmmune, Oxford, UK). Antibodies to detect protein phosphorylation were as follows: p-IRAK4 Thr345/Ser346 (Clone D6D7), IRAK4, p-S6-Alexa647 Ser235/236 (Clone D57.2.2E); p-SAPK/JNK Thr183/ Tyr185 (Clone 81E11), from Cell Signaling Technology. A Goat anti-Rabbit-Alexa647 secondary antibody (Invitrogen ${ }^{\mathrm{TM}}$ ) was used to stain p-IRAK4, IRAK4 and p-SAPK/ JNK. 
10. Eukaryotic cell lines

a. State the source of each eukaryotic cell line used.

b. Describe the method of cell line authentication used.

c. Report whether the cell lines were tested for mycoplasma contamination. of commonly misidentified cell lines maintained by ICLAC, provide a scientific rationale for their use. d. If any of the cell lines used are listed in the database

The following cell lines were used: 3-MCA derived mycoplasma-free sarcoma cell line MN/MCA1 and colon carcinoma cells (MC38).

MC38 cells were received from ATCC just before use.

MN/MCA1 cells were authenticated by checking morphology by microscopy after plating at different concentrations. A pathologist validated by histology sarcoma generated in vivo.

Cells were tested for Mycoplasma and only Mycoplasma free cells were used.

NA

\section{- Animals and human research participants}

Policy information about studies involving animals; when reporting animal research, follow the ARRIVE guidelines

\section{Description of research animals}

Provide details on animals and/or animal-derived materials used in the study.

All female and male mice used were on a C57BL/6J genetic background and 8-12 weeks-old, unless specified. Wild-type mice were obtained from Charles River Laboratories, Calco, Italy or were littermates of II1r8-/- mice. IL-1R8-deficient mice were generated as described35. ॥1r1-/- mice were purchased from The Jackson Labs, Bar Harbor ME, USA. All colonies were housed and bred in the SPF animal facility of Humanitas Clinical and Research Center in individually ventilated cages. I|1r1-/-/I1r8-/- mice were generated by crossing II1r1-/- and IIr8-/- mice. II18-/-/ \|1r8-/- were generated by crossing ॥18-/- and II1r8-/- mice.

Policy information about studies involving human research participants

\section{Description of human research participants}

Describe the covariate-relevant population characteristics of the human research participants.
Human peripheral mononuclear cells (PBMCs) were isolated from peripheral blood of 30-50-year old male and female healthy donors, upon approval by Humanitas Research Hospital Ethical Committee.

Human Bone Marrow mononuclear cells were collected from Humanitas Biobank, upon approval by Humanitas Research Hospital Ethical Committee (Authorization 1516, issued on February 26, 2016). 


\section{natureresearch}

Corresponding author(s): Cecilia Garlanda and Alberto Mantovani

\section{Flow Cytometry Reporting Summary}

Form fields will expand as needed. Please do not leave fields blank.

\section{- Data presentation}

For all flow cytometry data, confirm that:

$\bigotimes 1$. The axis labels state the marker and fluorochrome used (e.g. CD4-FITC).

$X^{2}$. The axis scales are clearly visible. Include numbers along axes only for bottom left plot of group (a 'group' is an analysis of identical markers).

$\bigotimes$ 3. All plots are contour plots with outliers or pseudocolor plots.

$\bigotimes 4$. A numerical value for number of cells or percentage (with statistics) is provided.

\section{- Methodological details}

5. Describe the sample preparation.

6. Identify the instrument used for data collection.

7. Describe the software used to collect and analyze the flow cytometry data.

8. Describe the abundance of the relevant cell populations within post-sort fractions.

9. Describe the gating strategy used.
Murine single-cell suspensions of BM, blood, spleen, lung and liver were obtained and stained. Human peripheral mononuclear cells (PBMCs) were isolated from peripheral blood of healthy donors, through a Ficoll density gradient centrifugation. Human Bone Marrow mononuclear cells were collected from Humanitas Biobank and thawed.

\section{BD LSRFortessa (TM) \\ BD FACSVerse (TM) \\ BD FACSAria (TM)}

FlowJo (9.3.2)

Post-sort cells were $99.37 \%+/-0.7 \%$ (SD) pure, as determined by reanalysing by FACS a fraction of sorted cells.

A relevant gating strategy for murine NK cells is described in Supplementary Figure 1

Tick this box to confirm that a figure exemplifying the gating strategy is provided in the Supplementary Information. $\bigotimes$ 